\title{
Effect of Loop Entropy on the Helix-Coil Transition of $\alpha$-Helical, Two-Chain, Coiled Coils
}

\author{
Jeffrey Skolnick* \\ Department of Chemistry, Louisiana State University, Baton Rouge, Louisiana 70803. \\ Received August 30, 1982
}

\begin{abstract}
The theory of the $\alpha$-helix to random coil transition in two-chain, coiled coils is extended to include the effect of loop entropy. To ascertain the conditions under which loop entropy is important, expressions are derived with the neglect of loop entropy for the ratio $(R)$ of the number of residues that are part of randomly coiled runs at the chain ends to the total number of randomly coiled residues. $R$ is derived for single chains $\left(R_{\mathrm{m}}\right)$ and for two-chain, coiled coil dimers $\left(R_{\mathrm{d}}\right)$ with and without coarse graining. When $R_{\mathrm{d}}$ lies near unity, there are few interior random coils, and the effect of loop entropy (which is important only for interior random coil sequences) can be neglected. In cases where loop entropy is important, we have extended Poland's recursion relation method (Biopolymers 1974, 13, 1859) to two-chain, coiled coils to incorporate loop entropy into the theory. For chains of moderate length, loop entropy is so severe as to eliminate loops entirely, producing a single interacting helical stretch in the dimer. Calculations with and without loop entropy are presented for the fraction of helix for dimers $\left(f_{\text {hd }}\right)$ as a function of the helix-helix interaction parameter $w$ and for the helix probability profiles in homopolymeric, two-chain, coiled coils. Where loop entropy cannot be neglected, it makes the helix-coil transition more cooperative and concomitantly modifies the helix probability profile. As expected, the interior of the dimer becomes more helical and the ends become less helical than if loop entropy is ignored.
\end{abstract}

\section{Introduction}

In a recent series of papers we have developed and applied a theory of the helix-coil transition in $\alpha$-helical, two-chain coiled coils (dimers) such as the muscle protein tropomyosin, Tm. ${ }^{1,2}$ The theory accounts for the enhanced stability of the parallel, in-register, side-by-side dimer of the two chains relative to the isolated single strands by a helix-helix interaction parameter $w$. The theory has been applied to a synthetic analogue of tropomyosin, $\mathrm{XY}_{5}^{2 \mathrm{a}}$ and has successfully explained the concentration dependence of the thermal denaturation profile of non-cross-linked Tm. So far, the qualitative picture presented by the realized theory (Scheraga's table of $\sigma$ and $s$ values for the amino acid residues in a single chain ${ }^{3-16}$ plus the two-chain, coiled coil, helix transition theory ${ }^{1}$ ) is in accord with the experimental data. ${ }^{2}$ However, the $w$ extracted from experiment must be viewed as a composite parameter characterizing the helix-helix interaction in that we have assumed $w$ is site independent and have not explicitly treated loop entropy. The former assumption can be alleviated by studying synthetic analogues of $T m$ such as $X_{5}$ that contain a repeating heptet; ${ }^{17}$ this is an experimental problem and does not reflect a fundamental limitation of the theory; the latter omission is intrinsic to the existing theory and must be addressed in greater detail. The primary goals of this paper are to provide a means of determining the conditions when loop entropy must be considered and, for those cases where loop entropy is important, to develop the formalism that incorporates loop entropy into the theory of two-chain $\alpha$-helix to random coil transitions. The result of such a development would be the removal of loop entropic effects from the values of $w$ obtained by fitting to experiment. Hitherto, such effects have been included willy-nilly in $w$.

Let us begin with a discussion of what loop entropy is, under what conditions it is important, and how it affects the helix content of a dimer. We shall consider noncross-linked chains unless otherwise specified. In the most general context, loop entropy is the reduction in configurational entropy of a sequence of $k$ randomly coiled residues constrained to have an end-to-end vector $\mathbf{r}$ relative

\footnotetext{
* Present address: Department of Chemistry, Washington University, St. Louis, MO 63130.
}

to the case where the ends are free. The reduction in entropy of closed loops, i.e., $\mathbf{r}=0$, is important in DNAtype double helices and also in singly stranded, poly (amino acids) that can exist in the hairpin configuration. ${ }^{18}$

There are two types of loop entropy encountered in two-chain, coiled coils. The first type, designated CC, is identical in functional form with that occurring in DNA. Suppose that the $i$ th residues on both chains are helical, i.e., an $\mathrm{h}(1) \mathrm{h}(2)$ configuration, and that the $i$ th helical pair is followed by $k$ consecutive random coil pairs $c(1) c(2)$ that terminate on another $\mathrm{h}(1) \mathrm{h}(2)$ pair. The $2 k$ random coil residues are thus constrained to form a closed loop. From the work of Schellman, ${ }^{19}$ Flory, ${ }^{20}$ and Jacobson and Stockmayer, ${ }^{21}$ we know that the statistical weight of the $2 k$ random coiled residues that form a ring is

$$
\delta_{1}(k)=C_{1} /(2 k+d)^{\alpha}
$$

The magnitude of the constant $C_{1}$ depends on the criterion of ring closure, i.e., how close the two ends must be before they are considered to form a closed ring; but, in any case, $C_{1} \ll 1$. The quantity $d$ accounts for the difficulty of forming a small ring in chains having fixed bond lengths, bond angles, and trans or gauche rotational potential minima. In a Gaussian chain, $\alpha=1.5$ and $d=0$. If excluded volume effects are included, $1.5<\alpha \leq 2 .{ }^{22,23}$

There is another kind of loop entropy that is entirely absent in a DNA-type helix but that can exist in a twochain, coiled coil. Suppose that residues $i$ and $i+k+1$ on chains one and two are helical and that one of the chains has $k$ consecutive helical residues while the neighboring chain contains $k$ consecutive random coil residues $i+1, \ldots, i+k$. Thus, these $k$ random coil residues are constrained to have an end-to-end vector $\mathbf{r}=k p / m$, with $p$ the pitch of the $\alpha$-helix (about $5.4 \AA /$ turn) and $m$ the number of residues per helical turn. We shall refer to these constrained open loops as $\mathrm{CH}$-type loops (for coil-helix). Defining $n \equiv k / m$, it is straightforward to show for a Gaussian chain that the statistical weight of the $k$ random coil residues with $|\mathbf{r}|=n p$ is

$$
\delta_{2}(n)=C_{2} n^{1 / 2} p^{2} l_{0}^{-3} \exp \left\{-3 / 2 n p^{2} l_{0}^{-2}\right\}
$$

with $l_{0}=m^{1 / 2} b_{0} ; b_{0}$ is the effective bond length per residue. $C_{2}$ is the factor that specifies the tolerance within which a vector $\mathbf{r}^{\prime}$ is said to be equal to $\mathbf{r}$. Of course, a random 
polypeptide chain is not a Gaussian coil and eq I-2 should be viewed as an approximate representation of the physics.

In addition to the cases discussed above, there is the possibility of nonzero, but unequal numbers of interior random coil sequences on the two strands. Clearly, this is a rather complicated case. Furthermore, given the uncertain validity of $\delta_{1}(n)$ and $\delta_{2}(n)$, it might seem to make a semiquantitative theory that includes loop entropy rather difficult. Fortunately, as we will demonstrate, this does not turn out to be the case.

Let $n_{\text {ERS }}$ be the average number of randomly coiled residues in sequences of randomly coiled residues that run from the ends of the chain, i.e., in end random sequences (ERS), and let $n_{\mathrm{IRS}}$ be the average number of randomly coiled residues in sequences of randomly coiled residues that are preceded and followed by at least one helical residue, i.e., an interior random sequence (IRS).

Clearly, the inclusion of loop entropy in the theory of two-chain, coiled coils (dimers) will significantly modify the results obtained from a theory that ignores loop entropy only if

$$
R \equiv n_{\mathrm{ERS}} /\left(n_{\mathrm{ERS}}+n_{\mathrm{IRS}}\right)
$$

is appreciably less than unity. In the following, let $R_{\mathrm{d}}$ refer to dimers and $R_{\mathrm{m}}$ to monomers (single chains). Two factors determine whether interior random coils in dimers are likely in a treatment that neglects loop entropy: the total number of residues in the molecule, $N$, and the helix initiation parameter $\sigma .^{24}$ If the molecule is sufficiently short, the randomizing effect of the ends leads to an $R_{\mathrm{d}}$ or $R_{\mathrm{m}}$ near unity; thus, essentially all unwinding is from the ends.

Consider now a chain of moderate length; whether or not interior random coils occur depends on the magnitude of $\sigma .^{22}$ Small values of $\sigma$ indicate that helix-coil boundaries are unlikely and $R$ will lie near one. As $\sigma$ increases, it becomes easier to form helix-coil boundaries, and the average number of helical sequences in the molecule becomes greater than one. Thus interior random coils become more common, and it is in this regime that loop entropy would be expected to be important in the dimers.

In the infinite-chain limit, $R=0$; i.e., there are no ends and all random residues are part of interior random sequences. The incorporation of loop entropy in this regime will exert a drastic influence on the behavior of the helix-coil transition and will serve to make it very cooperative.

The $\alpha$-helical, two-chain, coiled coils of experimental interest are in the small to moderate length regime. Short chains will have $R_{\mathrm{d}}$ (calculated with the neglect of loop entropy) near one. Thus loop entropy can, as indicated above, be neglected. However, in chains of moderate length such as tropomyosin, loop entropy can possibly exert an important effect by decreasing the average number of interior random coils in the molecule; i.e., $R_{\mathrm{d}}$ is increased. In other words, loop entropy acts to decrease the effective value of the helix initiation parameter. In fact, as shown in section IV, loop entropy acts to essentially set the statistical weight of an interior random coil in the interacting helical stretches equal to zero. In other words, interior random coils do not occur in the interacting portions of two-chain, coiled coils. The decreased effective value of $\sigma$ makes the helix-coil transition more cooperative. If the helix content of the dimer $\left(f_{\text {hd }}\right)$ is greater than (less than) $50 \%$, the value of $w$ necessary to give that helix content using an neglect-loop-entropy theory is larger (smaller) than would be obtained if an include-loop-entropy theory were used.

This is not the full story, however. The discussion to this point is applicable to DNA-type helices as well as to

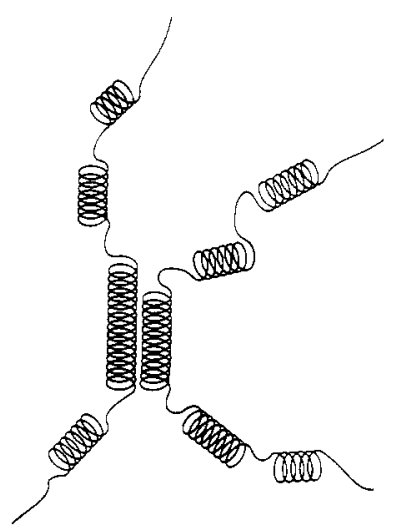

Figure 1. Example of a typical configuration in a two-chain, coiled coil in which interior random coils have been excluded from the interacting parts of the molecule.

two-chain, coiled coils. Yet, there is a fundamental difference between the DNA helix and a two-chain, side-byside pair of $\alpha$-helices. To have a helical conformation in DNA requires two strands; the statistical weight of a helical residue occurring on only one of the two strands is zero; i.e., it does not happen. In an $\alpha$-helical, two-chain, coiled coil, the statistical weight of a helical residue on one of the two chains is not zero. Thus, while interior random coils will not occur in those portions of the dimer that are side by side and interacting, one can conceivably have an interacting sequence of helical pairs $h(1) h(2)$ that is terminated by a random coil stretch on each chain and then followed by two helical, but noninteracting, and presumably separated, strands. Loop entropy acts to exclude interior random coils when bounded by interacting helices in the molecule but has no effect if the strands are separated. (See Figure 1.)

The partition function constructed for a two-chain, coiled coil with loop entropy must include the following: In a given conformation there is a single interacting helical stretch possibly preceded and followed by noninteracting parts of the chains that arises after a $c(1) c(2)$ or $c(1) h(2)$ (or equivalently an $\mathrm{h}(1) \mathrm{c}(2)$ ) configuration occurs. The noninteracting portions of the two-chains may possess interior random coil sequences. Thus, the specific form of the loop entropy is not in fact required; we must merely recognize that the loop entropy that would accompany the presence of interior coils induces partial strand separation and thereby eliminates the helix-helix interaction. This is the fundamental difference between the loop entropy effect in DNA and in two-chain, coiled coils of moderate length.

Tropomyosin may be cross-linked by oxidation of the cysteine- 190 residues. The presence of a cross-link modifies $\sigma$ and $s$ of the cysteine and also introduces a local stress in the $\alpha$-helices of the two-chain, coiled coil. Hence, cross-linking acts to destabilize the cross-linked dimer relative to the non-cross-linked dimer. This is seen in the difference between the experimental thermal denaturation curve of cross-linked Tm and the theoretical curve of the non-cross-linked dimer. ${ }^{2 \mathrm{a}}$ Recently, Mattice and Skolnick devised a theory for cross-linked chains that postulates that the interacting helices zip up from the cross-link. ${ }^{25}$ This treatment incorporates loop entropy in essentially the same manner as that discussed above for non-cross-linked chains, the only difference being that the interacting helices in a cross-linked chain must include the region of the cross-link, whereas in a non-cross-linked chain the interacting helical stretch can occur anywhere in the dimer. We recognize that alternative theories of the helix-coil transition in cross-linked chains that explicitly incorporate 
loop entropy may be required. Because of the uncertainty in $\sigma$ and $s$ of the cross-linked cysteine residue, we believe the problem of loop entropy in cross-linked chains should be addressed more fully at a later date. In the rest of the paper, we turn our attention to the simpler system: the non-cross-linked, two-chain, coiled coil.

The remainder of the paper provides the detailed theoretical basis for the preceding discussion of loop entropy. Section II reviews the coarse graining approximation and other salient aspects of the original method, which neglects loop entropy. Section III presents the calculation of $R$ for single chains and for two-chain, coiled coils with the neglect of loop entropy. Then section IV presents the extension of Poland's recursion relation method for probabilities to include two-chain, coiled coils, with and without loop entropy, and application is made to homopolymers. Section $\mathrm{V}$ summarizes the results and discusses possible future directions of research.

\section{Summary of Previous Theory Neglecting Loop Entropy}

An analysis of the primary sequence of tropomyosin reveals a quasi-repeating heptet designated by letters ag. ${ }^{26-29}$ Positions a and $\mathrm{d}$ tend to be occupied by hydrophobic residues, position e tends to be anionic, and position $\mathrm{g}$ tends to be cationic. In order for residues $\mathrm{a}$ and $\mathrm{d}$ to be in contact with their hydrophobic counterparts $\left(a^{\prime}\right.$ and $\left.d^{\prime}\right)$ on the adjacent chain, residues a- $d$ and $a^{\prime}-d^{\prime}$ must all be helical. Similarly, in order to accommodate the possibility of salt bridge formation, residues $\mathrm{e}-\mathrm{g}$ on both chains must also be helical. To incorporate this essential physics into the partition function, the coarse graining approximation, originally introduced by Crothers and Kallenbach, is employed. ${ }^{30}$ An outline of the method is presented below.

Consider a heteropolymeric chain containing $N T$ residues divided into $N$ blocks containing $m$ residues per block. Let $\mathrm{H}(\mathrm{C})$ denote a helical (random coil) block, i.e., a block composed of $m$ successive helical (random coil) residues, and let $[\mathrm{C}] \mathrm{H}$ and $[\mathrm{H}] \mathrm{C}$ denote a helical block and coil block at the interface of a stretch of coil and helical blocks, respectively. The statistical weights of the various conformations associated with the $i$ th block are as follows:

$\begin{array}{cl}\begin{array}{c}\text { conformation } \\ {[\mathrm{C}] \mathrm{C}}\end{array} & \text { statistical weight } \\ {[\mathrm{H}] \mathrm{H}} & 1 \\ {[\mathrm{C}] \mathrm{H}} & S M_{i}=\prod_{j=1}^{m} s_{j} \\ & \tau_{i}=\sum_{j=1}^{m} \sigma_{j} \prod_{k=j}^{m} s_{k} \\ {[\mathrm{H}] \mathrm{C}} & \mathcal{S}_{i}=1+\sum_{j=1}^{m-3} \prod_{k=1}^{j} s_{k}\end{array}$

with $\sigma$ and $s$ the standard helix initiation and propagation parameters. ${ }^{24}$ For a homopolymer, $\mathcal{f}=\left(s^{m}-1\right) /(s-1)$ and $\tau=\sigma s \mathcal{H}$. The treatment may be readily extended to chains composed of nonidentical-sized blocks. In particular, to accommodate the quasi-repeating heptet characteristic of tropomyosin, we employed alternating four- $(\mathrm{a}-\mathrm{d})$ and three- $(\mathrm{e}-\mathrm{g})$ residue blocks.

The statistical weight matrix of the ith block in an isolated, single chain may be written as

$$
\mathrm{U}_{i}=\stackrel{\mathrm{C}}{\mathrm{H}}\left[\begin{array}{cc}
1 & \mathrm{H} \\
\mathfrak{S}_{i} & S M_{i}
\end{array}\right]
$$

Now the statistical weight matrix of the $i$ th pair of interacting blocks in the two-chain, coiled coil may be expressed as

$$
\mathbf{U}_{\mathrm{d} i}=\left(\mathbf{U}_{i, 1} \otimes \mathbf{U}_{i, 2}\right) \mathbf{E}_{w}
$$

1 and 2 label the chains, $\otimes$ denotes the direct product, and $\mathbf{E}_{w}$ is a diagonal matrix with unity everywhere along the diagonal except for $E_{w}(4,4)=w . w$ is the helix-helix interaction parameter and is the ratio of the statistical weight of the interacting helical block pair in the dimer to the statistical weight of a noninteracting pair of helical blocks in the dimer. Equation II-3 implies that the statistical weight of a randomly coiled $[\mathrm{C}] \mathrm{C}$ and $[\mathrm{H}] \mathrm{C}$ is one irrespective of the location of the random coil (interior sequence vs. terminal sequence).

Following Flory, ${ }^{31}$ we can construct the internal partition function, $Z_{\text {sd }}$, for the two-chain, coiled coil by

$$
Z_{\text {sd }}=\operatorname{Row}(1,0,0,0) \prod_{i=1}^{N} \mathbf{U}_{\mathrm{d} i} \operatorname{Col}(1,1,1,1)
$$

Row $(\cdots)$ and Col (...) denote a row and column vector, respectively. Furthermore, the mean helix content in the two-chain, coiled coil can be obtained from ${ }^{1}$

$$
f_{\mathrm{hd}}=\operatorname{Row}(1,0,0,0,0,0,0,0) \prod_{i=1}^{N} \mathbf{A}_{i} \operatorname{Col}(0,0,0,0,1,1,1,1)
$$

with $\mathbf{A}_{i}$ an $8 \times 8$ supermatrix of the form

$$
\mathbf{A}_{i}=\left[\begin{array}{c:c}
\mathbf{U}_{\mathrm{d} i} & \mathbf{U}^{\prime} \mathrm{d} i \\
\hdashline 0 & \mathrm{U}_{\mathrm{d} i}
\end{array}\right]
$$

0 is a $4 \times 4$ null matrix and

$$
\mathbf{U}_{d}^{\prime}=\left(\mathbf{U}_{i, 1} \otimes \sum_{j=1}^{m} \frac{\partial \mathbf{U}_{i, 2}}{\partial \ln s_{j}}\right) \mathbf{E}_{w}
$$

In writing eq II-5a to II-6, we have assumed both chains of the two-chain, coiled coil are identical; extension to nonidentical chains is straightforward.

The theory presented here for $f_{\text {hd }}$ has been applied to extract $w(T)$ from experiment for a synthetic analogue of $\mathrm{Tm}, \mathrm{XY}_{5}{ }^{2 \mathrm{a}}$ and for tropomyosin at near-neutral $\mathrm{pH}^{2 \mathrm{~b}}$ and at acidic $\mathrm{pH} .{ }^{32}$

\section{Fraction of Random Residues in Terminal Sequences}

The existing (neglect of loop entropy) theory of the helix-coil transition in two-chain, coiled coils can provide an estimate of when the neglect of loop entropy is a valid approximation. In the following the superscript zero refers to quantities obtained with the neglect of loop entropy theory. Define $N_{\text {ERS }}$ as the average number of random residues located in end random sequences and $N_{c}$ as the total number of random residues. Thus, the fraction of residues that are both random and in end sequences is

$$
f_{\mathrm{ce}}{ }^{0}=N_{\mathrm{ERS}} / N
$$

and the total fraction of random residues is

$$
f_{\mathrm{c}}=1-f_{\mathrm{hd}}=N_{\mathrm{c}} / N
$$

Of utmost importance in estimating when loop entropy can be neglected is

$$
R=f_{\mathrm{ce}}{ }^{0} / f_{\mathrm{c}}=N_{\mathrm{ERS}} / N_{\mathrm{c}}
$$

Loop entropy acts only on interior random coils. If without consideration of loop entropy there are essentially no in- 
terior random coils anyway, that is, $R_{\mathrm{d}} \simeq 1$, then the neglect of loop entropy is entirely justified. Under these circumstances, values of $w(T)$ obtained from the original theory will be very close to if not identical with values extracted from a theory that includes loop entropy.

Poland and Scheraga have presented expressions for $f_{\mathrm{ce}}{ }^{0}$ of an isolated single homopolypeptide chain ${ }^{22}$ based on the $3 \times 3$ matrix method of Lifson and Roig. ${ }^{33}$ In this section we shall derive expressions for $f_{\mathrm{ce}}{ }^{0}$ in the $m=1$ approximation and in the coarse graining approximation for single heteropolymeric chains. Both a matrix expression and a serial matrix product expression for $f_{\mathrm{ce}}{ }^{0}$ will be derived. We shall then extend the treatment to $\alpha$-helical, two-chain, coiled coils. Application will be made to homopolymers and to rabbit $\alpha$-tropomyosin.

$m=1$ Approximation for Single Chains. The expression for $f_{\text {mce }}$ in a single chain in the Zimm-Bragg, $m$ $=1$ approximation is simplest to obtain, and thus we derive $f_{\text {mce }}$ for this case first. Let $l$ be the length (in residues) of a helical stretch, i.e., a sequence whose first and last residues are in the helical conformation. (Residues 2...l-1 may adopt any conformation.) Then

$$
\begin{array}{r}
f_{\text {mce }}=\left(N+\sum_{l=1}^{N-1} \sum_{n=0}^{N-l}(N-l) J *\left[\begin{array}{ll}
0 & \sigma s_{n+1} \\
0 & 0
\end{array}\right] \prod_{k=2}^{l-1} U_{n+k} .\right. \\
\left.\left[\begin{array}{cc}
0 & \sigma s_{n+l} \\
0 & s_{n+l}
\end{array}\right]\left[\begin{array}{cc}
0 & 0 \\
1 & 0
\end{array}\right] J\right) / N Z_{\mathrm{mc}}
\end{array}
$$

where $\boldsymbol{J} *=$ Row $(1,0), \boldsymbol{J}=\mathrm{Col}(1,1)$, and $Z_{\mathrm{mc}}$ is the partition function of a single chain; since $m=1, N T=N$. The first matrix in the numerator of eq III- 4 specifies that residues $1 \ldots n$ are random coil states and that residue $n+$ 1 is in a helical state. The matrix product allows for all possible states in residues $n+2 \ldots n+l-1$. The third matrix specifies residue $n+l$ as a helical state. The fourth matrix gives residues $n+l+1 \ldots N$ as random coiled states.

An expression equivalent to eq III-4 that is computationally more efficient and based on a serial matrix product may be written as

$$
\begin{aligned}
f_{\mathrm{mce}}=\left\{[1,0,0,0] \prod_{i=1}^{N} \mathbf{A}_{\mathrm{fi}} \mathrm{Col}(0,0,1,1)+\right. \\
{\left.[1,0,0,0] \prod_{i=1}^{N} \mathbf{A}_{\mathrm{bi}} \mathrm{Col}(0,0,1,1)-N\right\} / N Z_{\mathrm{mc}} } \\
\mathrm{A}_{\mathrm{f}}=\left[\begin{array}{ccc}
\mathrm{U}_{\mathrm{f}} & \mathrm{U}_{\mathrm{f}} \\
\hdashline 0 & \mathrm{U}
\end{array}\right] \quad \mathrm{A}_{\mathrm{b}}=\left[\begin{array}{c:c}
\mathrm{U} & \mathrm{U}_{\mathrm{b}} \\
\hdashline 0 & \mathrm{U}_{\mathrm{b}}
\end{array}\right]
\end{aligned}
$$

with $\mathrm{U}_{i}$ given by eq II-2 with $m=1$, and

$$
\mathrm{U}_{\mathrm{f}}=\left[\begin{array}{ll}
1 & 0 \\
0 & 0
\end{array}\right] \quad \mathrm{U}_{\mathrm{b}}=\left[\begin{array}{ll}
1 & 0 \\
1 & 0
\end{array}\right]
$$

The first (second) class of terms in eq III- 5 generates the contribution to $f_{\text {mce }}$ of all those sequences which unwind from the left-hand (right-hand) side of the molecule, and the third term corrects for overcounting of the all-random-coil state. We have applied eq III-4 and III-5 to a homopolymeric, two-chain, coiled coil with $N=284, \sigma=$ $4.7 \times 10^{-4}$, and $s=0.94 t$, with $1.0 \leq t \leq 1.40$. The helix content varies from 0.082 to 0.972 . The values of $R_{\mathrm{m}}$ (equivalently $f_{\text {mce }}$ ) calculated from the two methods agree to nine significant figures, thereby providing numerical verification of the equivalence of eq III-4 and III-5.

$f_{\text {mce }}$ in Single Chains with Coarse Graining. The coarse graining approximation introduces minor modifications of the expressions in eq III-4 and III-5. We begin with a modification of eq III-4.

$$
\begin{aligned}
& f_{\text {mce }}=\left\{N m+\sum_{l=1}^{N} \sum_{n=0}^{N-l}\left[\begin{array}{ll}
\tau^{\prime} \\
u
\end{array}\right] \prod_{k=2}^{l-1} \mathrm{U}_{n+k}\left[\begin{array}{ll}
0 & \tau_{n+l}{ }^{\alpha} \\
0 & S M_{n+l}{ }^{\alpha}
\end{array}\right] \bullet\right.
\end{aligned}
$$

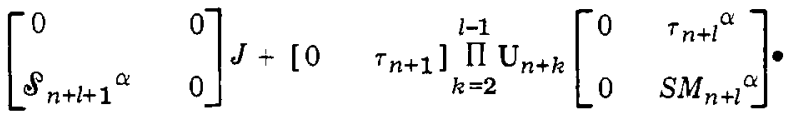

$$
\begin{aligned}
& \left.\left[\begin{array}{ll}
0 & 0 \\
\rho_{n+l+1} & 0
\end{array}\right] J\right\} / N m Z_{\mathrm{mc}}
\end{aligned}
$$

with

$$
\begin{gathered}
\tau_{n+l^{\alpha}}=\tau_{n+l} \quad \text { if } l>1 \\
=1 \quad \text { if } l=1 \\
S M_{n+l}{ }^{\alpha}=S M \quad \text { if } l>1 \\
=1 \quad \text { if } l=1 \\
S_{n+l+1}^{\alpha}=\mathcal{S} \quad \text { if } n+l+1 \leq N \\
=1 \quad \text { if } n+l+1>N
\end{gathered}
$$

and

$$
\prod_{k=2}^{l-1} \mathbf{U}_{n+k}=\mathbf{E} \quad \text { if } l<2
$$

Furthermore, with the $\tau$ defined in eq II-1,

$$
\tau_{u}^{\prime}=\sum_{j=1}^{m}(n m+j-1) \sigma_{j} \prod_{k=j}^{m} s_{k}
$$

is the number of random coils at the left end of the molecule when residues $1 . . . n$ are coil blocks and residue $n+1$ is a $[\mathrm{C}] \mathrm{H}$ block multiplied times the statistical weight of the particular conformation having $j-1$ coils in the [C]H block. Equation III-8 reduces to eq III-4 if $m=1$. Similarly, with $\mathcal{S}$ defined in eq II-1

$$
\left.\mathcal{S}^{\prime}{ }_{n+l+1}^{\alpha}=m(N-n-l)+\sum_{j=1}^{m-1}[(N-n-l) m-j)\right] \prod_{k=1}^{j} s_{k}
$$

is the number of coils occurring to the right of an $[\mathrm{H}] \mathrm{C}$ block at residue $n+l+1$ followed by coiled blocks $n+$ $l+2 \ldots . N$ times the statistical weight of blocks $n+l+1 \ldots . N$ associated with this end-coil sequence.

Observe that in eq III- 8 the all-helix state is included since $\tau$ contains some random coil states and that eq III-8 contains a common factor of $m$ in each expression. For a chain containing blocks of identical size, we can divide out $m$. This gives for the mean fraction of randomly coiled blocks that unwind from the ends

$$
f_{\text {mce }}=\left\{N+\sum_{l=1}^{N} \sum_{n=0}^{N-l}\left[0 \quad \tau_{\mathrm{b}}^{\prime}\right]_{k=2}^{l-1} \mathrm{U}_{n+k}\left[\begin{array}{ll}
0 & \tau_{n+l}{ }^{\alpha} \\
0 & S M_{n+l}^{\alpha}
\end{array}\right]\right. \text {. }
$$

$\left[\begin{array}{ll}0 & 0 \\ \rho_{n+l+1}^{\alpha} & 0\end{array}\right] J+[0$

$$
\left.\tau_{n+1}\right] \prod_{k=2}^{l-1} \mathrm{U}_{n+k}\left[\begin{array}{ll}
0 & \tau_{n+l}^{\alpha} \\
0 & S M_{n+l}^{\alpha}
\end{array}\right]
$$

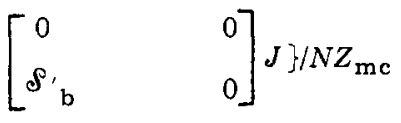

with

$$
\begin{gathered}
\tau_{\mathrm{b}}^{\prime}=\tau_{\mathrm{u} / m}^{\prime} \\
\mathcal{S}_{\mathrm{b}}^{\prime}=\mathcal{S}_{n+l+1 / m}^{\alpha}
\end{gathered}
$$

For a chain containing blocks of varying sizes, eq III-12a is still the mean number of random coiled blocks on ends but eq III-8 and III-12a are no longer equivalent. 
Setting $m=4, N=71, \sigma=4.7 \times 10^{-4}, s=0.94$, and $w$ $=1$, we find $R_{\mathrm{m}}$ calculated with eq III- 8 is 0.829 . In the $m=1$ approximation, $R_{\mathrm{m}}=0.799$, excellent agreement. By way of comparison, $f_{\mathrm{hd}}{ }^{0}=0.816$ and 0.823 for the same chain with and without coarse graining.

The matrix serial product expression that is equivalent to eq III-12a is

$$
\begin{gathered}
f_{\text {mce }}=\left\{\operatorname{Row}(1,0,0,0) \prod_{i=1}^{N} \mathbf{A}_{\mathrm{fc}, i} \operatorname{Col}(0,0,1,1)+\right. \\
\left.\operatorname{Row}(1,0,0,0) \prod_{i=1}^{N} \mathbf{A}_{\mathrm{bc}, i} \operatorname{Col}(0,0,1,1)-N\right\} / N Z_{\mathrm{mc}}
\end{gathered}
$$

where

$$
\begin{aligned}
& \mathrm{A}_{\mathrm{fc}, i}=\left[\begin{array}{c:c}
\mathrm{U}_{\mathrm{fc}_{\mathrm{c}}} & \mathrm{U}_{\mathrm{fc}_{\mathrm{c}}} \\
\hdashline 0 & \mathrm{U}
\end{array}\right]_{i} \\
& \mathrm{~A}_{\mathrm{bc}, i}=\left[\begin{array}{ccc}
\mathrm{U} & 1 & \mathrm{U}_{\mathrm{bc}} \\
\hdashline 0 & \mathrm{U}_{\mathrm{bc}}
\end{array}\right]_{i}
\end{aligned}
$$

with

$$
\mathrm{U}_{\mathrm{f} c}=\left[\begin{array}{cc}
1 & \tau_{\mathrm{p}} \\
0 & 0
\end{array}\right]
$$

and

$$
\tau_{\mathrm{p}}=\sum_{j=1}^{m}\left(\frac{j-1}{m}\right) \sigma_{j} \prod_{k=j}^{m} s_{k}
$$

Similarly

$$
\begin{gathered}
\mathrm{U}_{\mathrm{bc}}=\left[\begin{array}{ll}
1 & 0 \\
\mathcal{S}_{\mathrm{p}} & 0
\end{array}\right] \\
\mathcal{S}_{\mathrm{p}}=1+\sum_{j=1}^{m-1}\left(\frac{m-j}{m}\right) \prod_{k=1}^{j} s_{k}
\end{gathered}
$$

Setting $m=1$ in eq III-15 and III-16, we find that eq III-13 reduces to eq III-5.

$\boldsymbol{f}_{\mathrm{dce}}{ }^{0}$ in Two-Chain, Coiled Coils. We are now in a position to construct the expression for $f_{\mathrm{dce}}{ }^{0}$ in a coiled coil. The essentials of the analysis are illustrated by the following simple example. Suppose we know $P_{\mathrm{AB}}$, the statistical weight of the state $\mathrm{AB}$ and we want $q_{\mathrm{A}}$, the statistical weight of state $A$, without regard to state $B$. Clearly

$$
q_{\mathrm{A}}=\sum_{\mathrm{B}} P_{\mathrm{AB}}
$$

This is precisely the problem faced when calculating $f_{\mathrm{dce}}{ }^{0}$ in the dimers. We shall require the statistical weight of a coil or helical block on one of the chains given that the two chains are not independent.

Thus an expression analogous to eq III-12a for the fraction of random coils that unwind from the ends in a two-chain coiled coil, $f_{\mathrm{dce}}{ }^{0}$, can be derived. However, due to space limitations, we present here the computationally far more useful supermatrix expression for $f_{\mathrm{dce}}{ }^{0}$. Define

$$
\mathrm{U}_{\mathrm{fd}}=\left[\begin{array}{cc}
1 & \tau_{\mathrm{p}, 1} \\
0 & 0
\end{array}\right] \otimes\left[\begin{array}{cc}
1 & \tau_{2} \\
\mathcal{S}_{2} & S M_{2}
\end{array}\right] \mathrm{E}_{\mathrm{w}}
$$

with $\tau_{\mathrm{p}}$ defined in eq III-15b and

$$
\mathrm{U}_{\mathrm{bd}}=\left[\begin{array}{ll}
1 & 0 \\
\mathcal{S}_{\mathrm{p}, 1} & 0
\end{array}\right] \otimes\left[\begin{array}{ll}
1 & \tau_{2} \\
\mathfrak{S}_{2} & S M_{2}
\end{array}\right]
$$

with $\mathfrak{f}_{\mathrm{p}}$ defined in eq III-16b. Equations III-18 and III-19
Table I

Comparison of $f_{\mathrm{hd}}{ }^{\circ}$ and $R_{\mathrm{d}}{ }^{\circ}$ Obtained in the $m=1$ and $m=4$ Coarse Graining Approximation (Homopolypeptide, $n=284, s=0.94$, and $\sigma=3 \times 10^{-3}$ )

\begin{tabular}{lccccc}
\hline & \multicolumn{2}{c}{$m=1$} & & \multicolumn{2}{c}{$m=4$} \\
\cline { 2 - 3 } \cline { 5 - 6 }$w^{1 / m}$ & $f_{\mathrm{hd}}{ }^{0}$ & $R_{\mathrm{d}}{ }^{0}$ & & $f_{\mathrm{hd}}{ }^{0}$ & $R_{\mathrm{d}}{ }^{0}$ \\
\hline 1.000 & 0.2407 & 0.2989 & & 0.2378 & 0.3392 \\
1.024 & 0.2600 & 0.2915 & & 0.2571 & 0.3286 \\
1.047 & 0.2839 & 0.2846 & & 0.2811 & 0.3182 \\
1.068 & 0.3132 & 0.2785 & & 0.3105 & 0.3089 \\
1.088 & 0.3487 & 0.2737 & & 0.3462 & 0.3008 \\
1.107 & 0.3907 & 0.2708 & & 0.3882 & 0.2949 \\
1.125 & 0.4386 & 0.2703 & & 0.4360 & 0.2917 \\
1.142 & 0.4907 & 0.2727 & & 0.4879 & 0.2916 \\
1.158 & 0.5442 & 0.2780 & & 0.5411 & 0.2948 \\
1.174 & 0.5961 & 0.2862 & & 0.5929 & 0.3012 \\
1.189 & 0.6441 & 0.2968 & & 0.6408 & 0.3102 \\
1.204 & 0.6866 & 0.3090 & & 0.6833 & 0.3211 \\
1.218 & 0.7232 & 0.3223 & & 0.7201 & 0.3333 \\
1.231 & 0.7541 & 0.3359 & & 0.7513 & 0.3462 \\
1.245 & 0.7801 & 0.3495 & & 0.7776 & 0.3593 \\
1.257 & 0.8019 & 0.3628 & 0.7997 & 0.3722 \\
1.282 & 0.8356 & 0.3876 & 0.8340 & 0.3971 \\
1.305 & 0.8600 & 0.4099 & 0.8590 & 0.4198
\end{tabular}

indicate that $f_{\mathrm{dce}}{ }^{0}$ for chain 1 is being calculated. Equivalent results are obtained for identical chains when the order of the direct product is reversed. Then by analogy to eq III-13 we have

$$
\left\{\mathrm{J} * \prod_{i=1}^{N}\left[\begin{array}{cc}
\mathrm{U}_{\mathrm{fd}} & \mathrm{U}_{\mathrm{fd}} \\
3 & \mathrm{U}_{\mathrm{d}}
\end{array}\right] \mathrm{J}+\mathrm{J} * \prod_{i=1}^{N}\left[\begin{array}{ll}
\mathrm{U}_{\mathrm{d}} & \mathrm{U}_{\mathrm{bd}} \\
0 & \mathrm{U}_{\mathrm{bd}}
\end{array}\right] \mathrm{J}-N Z_{\mathrm{m}}\right\}
$$

$f_{\text {dce }}{ }^{0}=\frac{N Z_{\text {sd }}}{}$

(III-20)

with $\mathbf{J}^{*}=$ Row $(1,0,0,0,0,0,0,0), \mathbf{J}=\operatorname{Col}(0,0,0,0,1,1,1,1)$, and $Z_{\mathrm{mc}}$ the internal partition function of an isolated single chain. The first (second) class of terms generates all the average number random coils on one of the two chains that unwinds from the left-hand (right-hand) side of the molecule. The $-N Z_{\mathrm{m}}$ term corrects for overcounting of the all-random-coil state on one of the chains. Setting $w=1$ reduces eq III-20 to eq III-13. Equation III-20 is valid for all $m \geq 1$.

Calculations on Homopolypeptides. It is important to ascertain the validity of the coarse graining approximation used in eq III-20. In Tables I and II, $f_{\text {hd }}{ }^{0}$ and $R_{\mathrm{d}}{ }^{0}$ $=f_{\text {dce }}{ }^{0} /\left(1-f_{\text {hd }}{ }^{0}\right)$ were calculated in the $m=1$ and in the $m=4$ approximations for a two-chain homopolymer containing 284 residues per chain, with $s$ set equal to 0.94 and using eq II-5a and III-20. Values of $\sigma$ were $3 \times 10^{-3}$ (Table I) and $4.7 \times 10^{-4}$ (Table II). These choices give values of $R_{\mathrm{d}}{ }^{0}$ appreciably less than and near unity, respectively. In both cases, values of $w$ reported are normalized to the "per-residue value" i.e., $w^{1 / m}$. Clearly, in both cases $f_{\text {hd }}{ }^{0}$ calculated in the $m=1$ and $m=4$ approximations agree very well. The agreement between $R_{\mathrm{d}}{ }^{0}$ calculated in the $m=1$ and $m=4$ approximations is not as close as for $f_{\text {hd }}{ }^{0}$ but is still rather good; the behavior of $R_{\mathrm{d}}{ }^{0} \mathrm{vs} . w^{1 / m} \mathrm{cal}$ culated by both methods is the same. (The worst relative disagreement between the two methods is $14 \%$.) As $f_{\text {hd }}{ }^{0}$ $\rightarrow 1$, there are fewer $[\mathrm{C}] \mathrm{H}$ and $[\mathrm{H}] \mathrm{C}$ boundaries; thus, the $R_{\mathrm{d}}{ }^{0}$ calculated from the two methods converge. We therefore believe the coarse graining approximation can be used with confidence to calculate $R_{d}{ }^{0}$.

The occurrence of a minimum in $R_{\mathrm{d}}{ }^{0}$ as a function of $f_{\mathrm{hd}}{ }^{0}$ or equivalently $w$ is to be expected in a homopolymer. In the limit that $f_{\mathrm{hd}}{ }^{0} \rightarrow 0, R_{\mathrm{d}}{ }^{0} \rightarrow 1$; the all-random-coil state dominates. In the limit that $f_{\mathrm{hd}}{ }^{0} \rightarrow 1(w \rightarrow \infty)$, the hom- 
Table II

Comparison of $f_{\mathrm{hd}}{ }^{0}$ and $R_{\mathrm{d}}{ }^{0}$ Obtained in the $m=1$ and $m=4$ Coarse Graining Approximation (Homopolypeptide, $n=284, s=0.94$, and $\sigma=4.7 \times 10^{-4}$ )

\begin{tabular}{llllll}
\hline & \multicolumn{2}{c}{$m=1$} & \multicolumn{2}{c}{$m=4$} \\
\cline { 2 - 3 } \cline { 5 - 6 }$w^{-1 / m}$ & $f_{\mathrm{hd}^{0}}$ & $R_{\mathrm{d}}{ }^{0}$ & & $f_{\mathrm{hd}}{ }^{0}$ & $R_{\mathrm{d}}{ }^{0}$ \\
\hline 1.00 & 0.0824 & 0.7997 & 0.0815 & 0.8287 \\
1.024 & 0.0874 & 0.7923 & 0.0866 & 0.8216 \\
1.047 & 0.0952 & 0.7834 & 0.0944 & 0.8123 \\
1.068 & 0.1082 & 0.7717 & 0.1076 & 0.7998 \\
1.088 & 0.1321 & 0.7552 & 0.1317 & 0.7822 \\
1.107 & 0.1808 & 0.7325 & 0.1808 & 0.7579 \\
1.125 & 0.2824 & 0.7053 & 0.2828 & 0.7276 \\
1.142 & 0.4518 & 0.6870 & 0.4519 & 0.7047 \\
1.158 & 0.6194 & 0.6963 & 0.6188 & 0.7090 \\
1.174 & 0.7272 & 0.7234 & 0.7264 & 0.7328 \\
1.189 & 0.7901 & 0.7500 & 0.7895 & 0.7575 \\
1.204 & 0.8292 & 0.7711 & 0.8289 & 0.7776 \\
1.218 & 0.8555 & 0.7873 & 0.8555 & 0.7933 \\
1.231 & 0.8743 & 0.8002 & 0.8741 & 0.8060 \\
1.245 & 0.8883 & 0.8105 & 0.8882 & 0.8163 \\
1.257 & 0.8992 & 0.8192 & 0.8992 & 0.8250 \\
1.282 & 0.9151 & 0.8328 & 0.9151 & 0.8390 \\
1.305 & 0.9261 & 0.8432 & 0.9261 & 0.8499
\end{tabular}

opolymeric molecule will unwind solely from the ends and therefore $R_{\mathrm{d}}{ }^{0} \rightarrow 1$. Between the two limits of no helix and complete helix, the deviation from unity is determined by $\sigma$, and $R_{\mathrm{d}}{ }^{0}$ can develop a minimum. Comparison of Tables I and II shows that smaller values of $\sigma$ give larger values of $R_{\mathrm{d}}{ }^{0}$; i.e., it is much harder to form helix-coil boundaries, and unwinding from the ends dominates.

We have also reversed the order of the direct product in eq III-18 and III-19 and obtain nine significant figure agreement with $R_{\mathrm{d}}{ }^{0}$ shown in Table II.

The values of $\sigma$ and $s$ employed in Table II correspond to the "effective" $\sigma$ and $s$ values of tropomyosin at $30^{\circ} \mathrm{C}$ with $\sigma_{\text {eff }}=\left(\prod_{i=1}^{284} \sigma_{i}\right)^{1 / 284}$ and $s_{\text {eff }}=\left(\prod_{i=1}^{284} s_{i}\right)^{1 / 284}$. For the values of $R_{\mathrm{d}}{ }^{0}$ shown in Table II one would not expect loop entropy to be important in the homopolymeric analogue of tropomyosin; i.e., curves of $f_{\text {hd }}$ vs. $w$ calculated with and without loop entropy would be expected to lie fairly close to each other. However, this does not imply that loop entropy can be neglected in tropomyosin itself. The large variation in $\sigma$ and $s$ dictated by the primary sequence produces effects not mimicked by the homopolymeric analogue. We demonstrate this fact quantitatively below.

Application to Rabbit $\alpha$-Tropomyosin. In previous work Holtzer et al. have extracted $w(T)$ from experimental thermal denaturation curves of non-cross-linked rabbit $\alpha$-tropomyosin at near-neutral $\mathrm{pH}^{2 \mathrm{~b}}$ Adequate discussion of the analysis has been given elsewhere, and we view $w(T)$ as a known quantity throughout the course of the calculations presented below. (See eq 7 of ref $2 b$.)

Non-cross-linked tropomyosin is capable of undergoing dissociation into single chains. The overall helix content of the solution, $\Phi_{h}$, is given by

$$
\Phi_{\mathrm{h}}=f_{\mathrm{hm}} g_{\mathrm{m}}+f_{\mathrm{hd}}\left(1-g_{\mathrm{m}}\right)
$$

with $f_{\mathrm{hm}}$ and $g_{\mathrm{m}}$ the fraction of helix and weight fraction of single chains. In Table III we present $f_{\mathrm{hm}}$, and $R_{\mathrm{m}}=$ $f_{\text {mce }} /\left(1-f_{\mathrm{hm}}\right)$ as well as $f_{\mathrm{hd}}{ }^{0}$ and $R_{\mathrm{d}}{ }^{0}\left[=f_{\mathrm{dce}}{ }^{0} /\left(1-f_{\mathrm{hd}}{ }^{0}\right)\right]$ for tropomyosin in the 4,3 approximation from 20 to $80^{\circ} \mathrm{C}$ at $5^{\circ} \mathrm{C}$ intervals. The following observations are apparent:

(1) $R_{\mathrm{d}}{ }^{0}$, as calculated from this neglect-loop-entropy theory, differs significantly from unity and thus loop entropy may be important in tropomyosin. The fact that $R_{\mathrm{d}}{ }^{0}$ $<1$ is not at all surprising. A study of the helix probability profile reveals numerous maxima and minima, with
Table III

Tropomyosin in 4,3 Coarse Graining Approximation with the Neglect of Loop Entropy at Near-Neutral $\mathrm{pH}^{a}$

\begin{tabular}{lccccc}
\hline${ }^{\circ} \mathrm{C}$ & $f_{\mathrm{hm}}$ & $R_{\mathrm{m}}$ & $f_{\mathrm{hd}}{ }^{\circ}$ & $R_{\mathrm{d}}{ }^{0}$ & $\Phi_{\mathrm{h}}$ \\
\hline 20 & 0.2427 & 0.3797 & 0.9355 & 0.5111 & 0.9355 \\
25 & 0.2273 & 0.3962 & 0.9181 & 0.4793 & 0.9181 \\
30 & 0.2075 & 0.4186 & 0.8938 & 0.4532 & 0.8937 \\
35 & 0.1852 & 0.4464 & 0.8594 & 0.4382 & 0.8578 \\
40 & 0.1622 & 0.4792 & 0.8117 & 0.4384 & 0.7936 \\
45 & 0.1399 & 0.5161 & 0.7518 & 0.4533 & 0.6388 \\
50 & 0.1193 & 0.5559 & 0.6866 & 0.4778 & 0.3546 \\
55 & 0.1011 & 0.5971 & 0.6254 & 0.5054 & 0.1597 \\
60 & 0.0853 & 0.6383 & 0.5751 & 0.5331 & 0.1005 \\
65 & 0.0719 & 0.6782 & 0.5385 & 0.5601 & 0.0782 \\
70 & 0.0607 & 0.7158 & 0.5165 & 0.5864 & 0.0649 \\
75 & 0.0515 & 0.7504 & 0.5088 & 0.6123 & 0.0559 \\
80 & 0.0438 & 0.7816 & 0.5134 & 0.6377 & 0.0512
\end{tabular}

${ }^{a}$ The concentration of protein is $1 \times 10^{-5} \mathrm{~mol} / \mathrm{L}$.

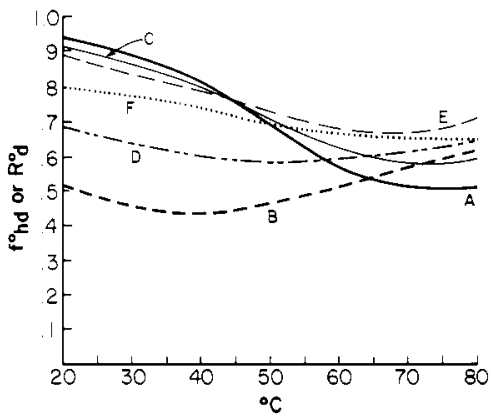

Figure 2. (A) $f_{\text {hd }}{ }^{0}$ in tropomyosin as a function of temperature (B) $R_{\mathrm{d}}{ }^{0}$ in tropomyosin as a function of temperature. (C) $f_{\mathrm{hd}}{ }^{0}$ in tropomyosin fragment residues 70-284 inclusive as a function of temperature. (D) $R_{d}{ }^{0}$ in tropomyosin fragment residues $70-284$ inclusive as a function of temperature. (E) $f_{\mathrm{hd}}{ }^{0}$ in tropomyosin fragment residues 70-214 inclusive as a function of temperature. (F) $R_{\mathrm{d}}{ }^{0}$ in tropomyosin fragment residues $70-214$ inclusive as a function of temperature.

prominent local minima near residues 55,214 , and 255 (see Figure 7 of ref 1). Therefore interior coil formation would be expected to be more important in tropomyosin than in the homopolymeric analogue, which has a flat probability profile (contrast Table II, column five, with Table III, column five). As the temperature is raised the less stable portions of tropomyosin are likely to melt first, leaving at higher temperatures the stable core. This conjecture is supported by the temperature dependence of $R_{\mathrm{d}}{ }^{0}$. (See also curve $\mathrm{B}$ of Figure 2 ; see below). $R_{\mathrm{d}}{ }^{0}$ initially decreases as the temperature increases (as the fraction of helix drops from one, $R_{\mathrm{d}}{ }^{0}$ must decrease). As $T$ increases, $R_{\mathrm{d}}{ }^{0}$ begins to rise again. This is conclusively demonstrated below, where several tropomyosin fragments are examined.

(2) $f_{\mathrm{hd}}{ }^{0}$ throughout the entire temperature range always exceeds 0.50 . This observation will prove important as a practical matter when we explicitly consider incorporation of loop entropy into the theory.

(3) $R_{\mathrm{m}}$ is an increasing function of $T$. For the single chain, we are always in the small-helix-content limit, and $R_{\mathrm{m}}$ must approach one as $f_{\mathrm{hm}}$ decreases, i.e., as $T$ increases.

We have also calculated $f_{\text {hd }}{ }^{0}$ in the $m=1$ approximation and find excellent agreement with $f_{\text {hd }}{ }^{0}$ calculated in the 4,3 approximation. Moreover, the absolute difference of $R_{\mathrm{d}}{ }^{0}$ calculated throughout the entire temperature range is less than 0.02 . The difference between the two methods decreases with increasing helix content.

In Figure 2, $f_{\mathrm{hd}}{ }^{0}$ and $R_{\mathrm{d}}{ }^{0}$ are plotted as a function of $T$ in curves $\mathrm{A}$ and $\mathrm{B}$, respectively, for the $\alpha$-tropomyosin dimer. $f_{\text {hd }}{ }^{0}$ and $R_{\mathrm{d}}{ }^{0}$ for a postulated two-chain tropomyosin fragment, Ar, residues 70-284, inclusive, are plotted vs. $T$ 
in curves $\mathrm{C}$ and $\mathrm{D}$. $f_{\mathrm{hd}}{ }^{0}$ (curve $\mathrm{E}$ ) and $R_{\mathrm{d}}{ }^{0}$ (curve $\mathrm{F}$ ) are shown as a function of temperature for a postulated twochain tropomyosin fragment, Am, residues 70-214, inclusive. All these curves are calculated in the $m=1$ approximation. The site-averaged $w$ appropriate for intact $\mathrm{Tm}$ at the given temperature was also used in the calculations of $R_{\mathrm{d}}{ }^{0}$ and $f_{\mathrm{hd}}{ }^{0}$ for the fragments $\mathrm{Ar}$ and Am. These calculations are for illustrative purposes only and are designed to elucidate the behavior of $R_{\mathrm{d}}{ }^{0} \mathrm{vs}$. $T$ in tropomyosin; they are not to be viewed as quantitative predictions for the Tm fragments. Indeed, these fragments have as yet not been isolated.

Observe the temperature dependence for Am, the fragment with the less stable regions excised; $R_{\mathrm{d}}{ }^{0}$ is a monotonically decreasing function of temperature. This is consistent with point 1 discussed above. $R_{\mathrm{d}}{ }^{0}$ in $\mathrm{Tm}$ and in $\mathrm{Ar}$ are concave-upward functions of $T$. We remind the reader that the helix probability profile of Tm contains local minima near residues 214 and 255. Thus Ar should have an unstable interior region near the $\mathrm{C}$ terminus. Clearly then, it is the presence of the less helical portions in the interior of the tropomyosin molecule that produces the appreciable number of interior random coils and therefore necessitates the incorporation of loop entropy into the treatment of the helix-coil transition of Tm.

Pato et al. have obtained various Tm fragments. ${ }^{34}$ In future work we shall extract $w(T)$ appropriate to these fragments. Depending on both the length and location of the excised portion, loop entropy may or may not be important.

We conclude this section with the observation that a theory which includes loop entropy must be developed if $w(T)$ extracted from thermal denaturation data of tropomyosin is to be viewed as a physically interpretable measure of the helix-helix interaction and not merely a broad composite that includes, among other things, a contribution from loop entropy. Moreover, if longer double- $\alpha$ helical molecules, such as paramyosin or myosin tails, are ever to be examined in the context of the developing theory, the incorporation of loop entropy into the treatment becomes a must. Section IV attempts to achieve this goal.

\section{Loop Entropy in Two-Chain, Coiled Coils}

In this section, we develop a method of treating the helix-coil transition in two-chain, coiled coils that includes loop entropy. We begin with an extension of Poland's recursion relation method for probability profiles to the two-chain, coiled coils that includes coarse graining but excludes loop entropy. ${ }^{35}$ This treatment is formally equivalent to the matrix method derived previously for two-chain, coiled coils" and is designated the "neglectloop-entropy" theory. Representative allowed conformations for the neglect-loop-entropy theory are $\alpha, \beta$, and $\delta$ and are shown in Figure 3A. States $\gamma$ and $\epsilon$ have been excluded because with the neglect of loop entropy these states have negligible statistical weight relative to the conformations $\beta$ and $\delta$, respectively. (Conformations $\gamma$ and $\epsilon$ have only one end that experiences an enhanced helix interaction whereas both ends in conformations $\beta$ and $\delta$ experience an enhanced helix-helix interaction.) We then focus on a simplified model, the "DNA-isomorphic" model (referred to in previous work as the $\mathrm{CC}-\mathrm{HH} \operatorname{model}^{1}$ ), to obtain a qualitative understanding of the effect of loop entropy on the helix-coil transition. In the DNA-isomorphic model, only $\mathrm{C}(1) \mathrm{C}(2)$ or interacting $\mathrm{H}(1) \mathrm{H}(2)$ block pairs are allowed, i.e., states $\alpha$ and $\beta$ of Figure 3B. The disallowed states $\gamma, \delta$, and $\epsilon$ are also shown for the DNA-isomorphic model in Figure 3B. The reader may

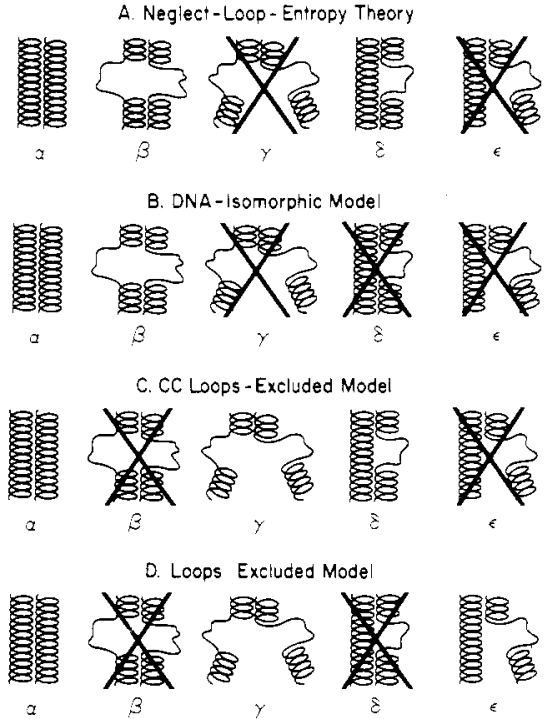

Figure 3. Allowed and disallowed (crossed out) sequences of internal random coils in (A) the previously derived neglectloop-entropy theory, (B) the DNA-isomorphic model, (C) the CC-loops-excluded model, and (D) the loops-excluded model. See text for a discussion of the various allowed and disallowed sequences.

recognize that as the name indicates the allowed types of states are isomorphic with a DNA double helix. Using the qualitative insight gained from the study of the DNA. isomorphic model, we then consider the effect of loop entropy in $\alpha$-helical, two-chain, coiled coils. A simplified treatment, the "CC-loops-excluded" model, is developed. In the CC-loops-excluded model, loop entropy is assumed to act on interior $\mathrm{C}(1) \mathrm{C}(2)$ type random coils and causes the exclusion of interior $\mathrm{C}(1) \mathrm{C}(2)$ coils in the interacting portion of the dimer. The allowed and disallowed conformations in the CC-loops-excluded model are shown schematically in Figure 3C. States $\alpha, \gamma$, and $\delta$ are permitted. State $\beta$ has been excluded due to the effect of loop entropy (no interior $\mathrm{CC}$ loops in the interacting portions of the dimer). State $\delta$ is included because by hypothesis loop entropy has been allowed to affect only interior $\mathrm{C}(1) \mathrm{C}(2)$ and not $\mathrm{C}(1) \mathrm{H}(2)$ (or equivalently $\mathrm{H}(1) \mathrm{C}(2)$ ) loops in the interacting regions of the dimer (an artificial assumption of the model). State $\epsilon$ is excluded because the statistical weight of state $\epsilon$ is negligible compared to state $\delta$ provided that loop entropy is ignored for interior $\mathrm{C}(1)$ $\mathrm{H}(2)$ type loops. Finally we present the "loops-excluded" model; representative allowed and disallowed conformations are shown in Figure 3D. In the loops-excluded model, loop entropy results in the exclusion of interior $\mathrm{C}(1) \mathrm{C}(2)$ or $\mathrm{C}(1) \mathrm{H}(2)$ type loops in the interacting portions of the dimer. Thus states $\beta$ and $\delta$ are forbidden while states $\alpha$, $\gamma$, and $\epsilon$ are allowed. As shown in states $\gamma$ and $\epsilon$, loop entropy results in a single interacting helical stretch possibly preceded or followed by random coiled stretches; any additional helical stretches that may occur are noninteracting. The loops-excluded model has also been depicted in Figure 1.

Extension of Poland's Treatment to Two-Chain, Coiled Coils. We shall essentially follow Poland's notation, with the substitution of $\mathrm{H}$, for helical block for his state " 1 ". ${ }^{35}$ Let $q_{\mathrm{R}}(n), q_{\mathrm{I}}\left(m_{\mathrm{I}}, n\right)$, and $q_{\mathrm{L}}(n)$ be the statistical weight of $n$ randomly coiled blocks occurring at the right end, interior, and left end, respectively, of one of the two strands in the molecule. We remind the reader that in this formulation the statistical weights $q_{\mathrm{R}}, q_{\mathrm{I}}$, and $q_{\mathrm{L}}$ are normalized to the all-helical conformation of one of the two strands in the molecule. Define $P\left(\mathrm{H}_{j} \mid \mathrm{H}\right)$ (Poland's $P\left(1_{j} \mid 1\right)$ ) 
as the conditional probability that the $(j+i)$ th block is helical if the $j$ th block is helical and $f_{\text {hd }}(j)$ as the probability that the $j$ th block is helical on one of the two chains (Poland's $P\left(1_{j}\right)$ ). Poland then proceeds to derive an expression for the $P\left(\mathrm{H}_{j} \mid \mathrm{H}\right)$ in terms of $P\left(\mathrm{H}_{k} \mid \mathrm{H}\right)$, with $k>j$, and $q_{\mathrm{R}}$ and $q_{\mathrm{I}}$. (See eq P4 and P5.) Furthermore, once the $P\left(\mathrm{H}_{j} \mid \mathrm{H}\right)$ are in hand, it is possible to calculate $f_{\text {hd }}(j)$ in terms of $q_{\mathrm{L}}, q_{\mathrm{I}}$, and the conditional helix probabilities. (See eq P9 and P10.)

The method also permits the calculation of $R_{\mathrm{d}}$ if eq P11 and $\mathrm{P} 19$ are modified to include the all-random-coil state. If $P(\mathrm{C} \mid N)$ is the probability that the all-random-coil state is occurring in one of the two strands, then

$$
q_{\mathrm{L}}(N)=P(\mathrm{C} \mid N) / f_{\mathrm{hd}}(1) \prod_{j=1}^{N-1} P\left(\mathrm{H}_{j} \mid \mathrm{H}\right)
$$

Thus the contribution to the average number of random coils on an end, $N_{\text {ERs }}$, due to the all-random-coil state of one of the chains is

$$
N q_{\mathrm{L}}(N) f_{\mathrm{hd}}(1) \prod_{j=1}^{N-1} P\left(\mathrm{H}_{j} \mid \mathrm{H}\right)=N P(\mathrm{C} \mid N)
$$

From the above it is apparent that the extension of Poland's method to two-chain, coiled coils is predicated on our ability to construct $q_{\mathrm{L}}, q_{\mathrm{I}}$, and $q_{\mathrm{R}}$. Once these statistical weights are in hand, the existing Poland formalism can be used straightforwardly.

Neglect of Loop Entropy Theory. We begin with the extension of Poland's theory to $\alpha$-helical, two-chain, coiled coils in which loop entropy is ignored. See Figure 3A. An equivalent matrix method has been derived in previous work. $^{1}$

Construction of $q_{\mathrm{R}}$ without Loop Entropy. Let $q_{\mathrm{R}}{ }^{0}(n)$ be the statistical weight of $n$ randomly coiled residues on the right end of one of the strands in which loop entropy is neglected. Consider now the following sequences on one of the two strands:

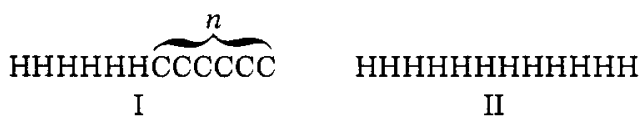

$Z_{\mathrm{I}}$, the statistical weight of I normalized with respect to a [C]C block of weight unity, is

$$
\begin{aligned}
& Z_{\mathrm{I}}=\operatorname{Row}(1,0,0,0)\left[\begin{array}{c:c}
0 & \tau_{1} \mathrm{U}_{1}(w) \\
\hdashline 0 & 0
\end{array}\right]\left[\begin{array}{c:c}
0 & 0 \\
\hdashline 0 & \prod_{i=2} S M_{i} \mathbf{U}_{i}(w)
\end{array}\right] .
\end{aligned}
$$

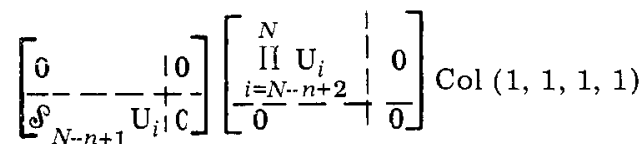

where

$$
\mathrm{U}_{i}(w)=\left[\begin{array}{ll}
1 & \tau_{i} w \\
\mathcal{f}_{i} & S M_{i} w
\end{array}\right]
$$

and $\mathbf{U}_{i}$ is given by eq II-2. (The dot indicates matrix multiplication.)

Performing all the indicated multiplications gives

$Z_{\mathrm{I}}=\tau_{1} \prod_{i=2}^{N-n} S M_{i} \mathcal{\rho}_{N-n+1} \boldsymbol{J} * \prod_{i=1}^{N-n} \mathbf{U}_{i}(w) \prod_{i=N-n+1}^{N} \mathbf{U}_{i} \boldsymbol{J}$

with $\boldsymbol{J} *=\operatorname{Row}(1,0)$ and $\boldsymbol{J}=\operatorname{Col}(1,1)$.

Similarly, the statistical weight of II is

$$
Z_{\mathrm{II}}=\tau_{1} \prod_{i=2}^{N} S M_{i} \boldsymbol{J} * \prod_{i=1}^{N} \mathbf{U}_{i}(w) \boldsymbol{J}
$$

Thus

$$
q_{\mathrm{R}}{ }^{0}(n)=\frac{Z_{\mathrm{I}}}{Z_{\mathrm{II}}}=\frac{\mathcal{S}_{N-n+1} \boldsymbol{J}^{*} \prod_{i=1}^{N-n} \mathbf{U}_{i}(w) \prod_{N-n+1}^{N} \mathbf{U}_{i} \boldsymbol{J}}{\prod_{i=N-n+1}^{N} S M_{i} Z_{\mathrm{w}}}
$$

with

$$
Z_{\mathrm{w}}=\boldsymbol{J} * \prod_{i=1}^{N} \mathrm{U}_{i}(w) \boldsymbol{J}
$$

That is, $Z_{\mathrm{w}}$ is the statistical weight of chain two when chain one is in the all-helix state.

Construction of $q_{L}$ without Loop Entropy. Let $q_{\mathrm{L}}{ }^{0}(n)$ be the statistical weight of the $n$-sequential randomly coiled blocks located on the left end of one of the two chains and in which loop entropy is ignored.

Consider then the sequence

\section{$\overbrace{\mathrm{CCCCC}}^{n} \mathrm{HHHH}$}

III

with statistical weight $Z_{\text {III }}$ normalized to the all-randomcoil state. If $n<N$

$$
Z_{\mathrm{III}}=\tau_{n+1} \prod_{i=n+2}^{N} S M_{i} \boldsymbol{e}^{*} \prod_{i=1}^{n} \mathbf{U}_{i} \prod_{i=n+1}^{N} \mathbf{U}_{i}(w) \boldsymbol{J} Z_{\mathbf{w}}{ }^{-1}
$$

and

$$
q_{\mathrm{L}}{ }^{0}(n)=\frac{Z_{\mathrm{III}}}{Z_{\mathrm{II}}}=\frac{\tau_{n+1} \boldsymbol{J}^{*} \prod_{i=1}^{n} \mathbf{U}_{i} \prod_{i=n+1}^{N} \mathbf{U}_{i}(w) \boldsymbol{J}}{\tau_{1} \prod_{i=2}^{n+1} S M_{i} Z_{\mathrm{w}}} \text { if } N>n
$$

Setting $n=N$, i.e., an all-random-coil sequence on one strand, we have

$$
q_{\mathrm{L}}(N)=\boldsymbol{J} * \prod_{i=1}^{N} \mathbf{U}_{i} \boldsymbol{J} /\left\{\tau_{1} \prod_{i=2}^{N} S M_{i} Z_{\mathrm{w}}\right\}
$$

Construction of $q_{I}^{0}\left(m_{I}, n\right)$ without Loop Entropy. Suppose blocks 1 to $m_{\mathrm{I}}$ on one of the two chains are helical, blocks $m_{\mathrm{I}}+1, \ldots, m_{\mathrm{I}}+n$ are random coils, and blocks $m_{\mathrm{I}}$ $+n+1, \ldots, N$ are helical blocks, viz.

$$
\overbrace{\mathrm{HHHH}}^{m_{\mathrm{I}}} \overbrace{\mathrm{CCCCC}}^{n} \overbrace{H H H H H}^{N-m_{\mathrm{I}}-n}
$$

IV

The statistical weight of IV normalized to the all-coil state is

$Z_{\mathrm{IV}}=$

$$
\begin{array}{r}
\tau_{1} \prod_{i=2}^{m_{1}} S M_{i} \mathcal{S}_{m_{\mathrm{I}}+1} \tau_{m_{1}+n+1} \prod_{i=m_{1}+n+2}^{N} S M_{i} \boldsymbol{J} * \prod_{i=1}^{m_{\mathrm{I}}} \mathbf{U}_{i}(w) \prod_{i=m_{\mathrm{I}}+1}^{m_{\mathrm{I}}+n} \mathbf{U}_{i} \\
\prod_{i=m_{\mathrm{I}}+n+1}^{N} \mathbf{U}_{i}(w) \boldsymbol{J}(\mathrm{IV}-12)
\end{array}
$$

Therefore

$$
\begin{aligned}
& q_{\mathrm{I}}^{0}\left(m_{\mathrm{I}}, n\right)=\frac{Z_{\mathrm{IV}}}{Z_{\mathrm{II}}}= \\
& \mathcal{S}_{m_{\mathrm{I}}+1} \tau_{m_{\mathrm{I}}+n+1} \boldsymbol{J} * \prod_{i=1}^{m_{\mathrm{I}}} \mathrm{U}_{i}(w) \prod_{i=m_{\mathrm{I}}+1}^{m_{\mathrm{I}}+n+1} \mathbf{U}_{i} \prod_{i=m_{\mathrm{I}}+n+2}^{N} \mathbf{U}_{i}(w) \boldsymbol{J} / \\
& \left\{\prod_{i=m_{\mathrm{I}}+1}^{m_{\mathrm{I}}+n+1} S M_{i} Z_{\mathrm{w}}\right\}(\mathrm{IV}-1)
\end{aligned}
$$

There is one further modification that must be made to Poland's treatment and that deals with the calculation of the internal partition function for the dimer, $Z_{\text {sd }}$. We 
prefer to normalize $Z_{\text {sd }}$ with respect to the all-random-coil configuration having a statistical weight of unity.

Now the probability of observing the all-helical sequence on one of the two strands is (see eq IV-6)

$$
\tau_{1} \prod_{i=2}^{N} S M_{i} Z_{\mathrm{w}} / Z_{\mathrm{sd}}=f_{\mathrm{hd}}(1) \prod_{j=1}^{N-1} P\left(\mathrm{H}_{j} \mid \mathrm{H}\right)
$$

or

$$
Z_{\mathrm{sd}}=\tau_{1} \prod_{i=2}^{N} S M_{i} Z_{\mathrm{w}} f_{\mathrm{hd}}(1) \prod_{j=1}^{N-1} P\left(\mathrm{H}_{j} \mid \mathrm{H}\right)
$$

A word of caution on the use of Poland's recursion relation method with coarse graining must be mentioned. When coarse graining is employed, the interfacial statistical weights $[\mathrm{C}] \mathrm{H}$ and $[\mathrm{H}] \mathrm{C}$ contain mixed helix and coil states. Thus $P\left(\mathrm{H}_{j} \mid \mathrm{H}\right)$ is not exact and certain small discrepancies between $f_{\text {hd }}$ calculated by this technique and the matrix method of section II may arise. Fortunately, in all cases employed here the differences between the two methods turn out to be fairly small, and especially in the range of interest where $f_{\text {hd }}$ is greater than $50 \%$, they can safely be ignored.

Suppose $w=1$, that is, the chains are independent and noninteracting; then the matrix products in the numerator and denominator of eq IV-7, IV-10, IV-11, and IV-13 cancel. It is then easy to show that Poland's equations for $q_{\mathrm{R}}{ }^{0}, q_{\mathrm{L}}{ }^{0}$, and $q_{\mathrm{I}}{ }^{0}$ are recovered (see eq P24).

DNA-Isomorphic Model. Consider the limiting case where we require the $i$ th block on both chains to be either helical, i.e., an $\mathrm{H}(1) \mathrm{H}(2)$ state, or randomly coiled, i.e., a $\mathrm{C}(1) \mathrm{C}(2)$ state. See Figure $3 \mathrm{~B}$. The equations for the $q_{\mathrm{L}}$, $q_{\mathrm{R}}$, and $q_{\mathrm{I}}$ can be obtained rather simply. Let

$$
\mathrm{U}_{i}(w)=\left[\begin{array}{ll}
0 & \tau w \\
0 & 0
\end{array}\right]_{i}
$$

for the statistical weight of a $[\mathrm{C}] \mathrm{H}$ block. Similarly, let

$$
\mathrm{U}_{i}(w)=\left[\begin{array}{ll}
0 & 0 \\
0 & S M w
\end{array}\right]_{i}
$$

for a helical block in the middle of a stretch of $\mathrm{H}(1) \mathrm{H}(2)$ block pairs and

$$
\mathbf{U}_{i}=\left[\begin{array}{ll}
1 & 0 \\
\rho & 0
\end{array}\right]_{i}
$$

everywhere a $\mathrm{C}(1) \mathrm{C}(2)$ pair occurs.

For convenience and brevity we present only the homopolymer case; extension to heteropolymers is obvious.

Employing the above statistical weight matrices in eq IV-8 gives

$$
Z_{\mathrm{w}}=\tau S M^{N-1} w^{N}
$$

and in eq IV -7 yields

$$
q_{\mathrm{R} i}(n)=\mathcal{f}^{2}\left(S M^{2} w\right)^{-n}
$$

Furthermore, from eq IV-10 if $n<N$

$$
q_{\mathrm{L} i}(n)=\left(S M^{2} w\right)^{-n}
$$

and from eq IV-11 if $n=N$

$$
q_{\mathrm{L}}(N)=1 /\left(\tau^{2}\left(S M^{2}\right)^{N-1} w^{N}\right)
$$

Finally eq IV -13 provides

$$
q_{\mathrm{I}}^{0}(m, n)=\tau^{2} \mathcal{S}^{2} w\left(S M^{2} w\right)^{-n-1}
$$

If loop entropy is included

$$
q_{\mathrm{I}}^{l}(m, n)=\tau^{2} \mathcal{S}^{2} w \delta(n) /\left(S M^{2} w\right)^{n+1}
$$

where

$$
\delta(n)=C_{1} /(2 m n+d)^{\alpha}
$$

$\delta(n)$ is the loop entropy associated with closed loops of the $\mathrm{CC}$ type in the coarse graining approximation. (Please see discussion immediately preceding and following eq I-1.) Since block sizes are small, fine graining of $\delta(n)$ is not required, as it is in DNA cases treated previously. ${ }^{22}$

To check the accuracy of the programing, we computed $f_{\text {hd }}{ }^{0}$ and $R_{\mathrm{d}}{ }^{0}$ with $\delta(n)=1$ (no loop entropy) for a 71-residue chain in the $m=1$ approximation with $\sigma=1 \times 10^{-3}$ and $s=0.94$ as a function of $w$ by Poland's method and using the standard matrix method as applied to a DNA-isomorphic model. Agreement to nine significant figures in $R_{\mathrm{d}}{ }^{0}$ and $f_{\mathrm{hd}}{ }^{0}$ was found. Since $R_{\mathrm{d}}$ is essentially unity for $w^{1 / m} \leq 1.488$, we set $\delta(n)=0$ (i.e., there are no internal loops); comparison of $f_{h d}$ with and without possibility of internal loop formation gave agreement to three significant figures throughout the calculated range of $w$, an entirely expected result.

Turning now to the coarse graining approximation, we set $N=71, m=4, \sigma=1 \times 10^{-4}$, and $s=0.94$ and calculate $f_{\text {hd }}$ and $R_{\mathrm{d}}$ vs. $w$ using Poland's method. Even in the absence of loop entropy, i.e., $\delta(n)=1, R_{\mathrm{d}}{ }^{0}>0.99$ throughout range $1 \leq w \leq 2.95$ ( $f_{\text {hd }}$ goes from $9.7 \times 10^{-6}$ to 0.95 ). Consequently, setting $\delta(n)=1 /(2 m n)^{1.5}, \delta(n)=0$ and $\delta(n)$ $=1$ gave values of $f_{\text {hd }}$ that agreed to at least three significant figures. Hence since there are essentially no interior random coils when $\delta(n)=1$, loop entropy is unimportant and can therefore safely be ignored.

Use of the matrix method (using eq 40 and the following equations of ref 1 ) gave three significant figure agreement with $f_{\text {hd }}$ calculated via the Poland method. The minor differences between the two techniques reflect the way $[\mathrm{H}] \mathrm{C}$ and $[\mathrm{C}] \mathrm{H}$ boundaries are handled in the two methods. Thus numerical differences between the two techniques are immaterial, and unless otherwise stated, we shall report results calculated via Poland's approach.

Suppose $\sigma$ is increased so that $R_{\mathrm{d}}{ }^{0}$ (calculated with the neglect of loop entropy and based on eq III-20 in the DNA-isomorphic model approximation) is significantly less than one; then we would expect $f_{\text {hd }}$ values calculated with and without loop entropy at a given $w$ to differ significantly.

In Figure 4 we have plotted $f_{\text {hd }}{ }^{0}$ and $R_{\mathrm{d}}{ }^{0}$ vs. $w$ for a 284-residue homopolymeric two-chain, DNA-isomorphic, coiled coil having $\sigma=7 \times 10^{-3}$ and $s=0.94$ in the $m=4$ coarse graining approximation. Curves $\mathrm{A}$ and $\mathrm{B}$ refer to $f_{\mathrm{hd}}{ }^{0}$ and $R_{\mathrm{d}}{ }^{0}$ calculated with $\delta(n)=1$, i.e., the approximation that the statistical weight of a $[\mathrm{C}(1) \mathrm{C}(2)] \mathrm{C}(1) \mathrm{C}(2)$ block and $[\mathrm{H}(1) \mathrm{H}(2)] \mathrm{C}(1) \mathrm{C}(2)$ block are 1 and $\mathcal{f}^{2}$, respectively, independent of the location of the random coil (no loop entropy). Curves $\mathrm{C}$ and $\mathrm{D}$ show $f_{\mathrm{hd}}$ and $R_{\mathrm{d}}$ vs. $w$ when $q_{\mathrm{I}}\left(m_{\mathrm{I}}, n\right)=0.0$; i.e., loop entropy is so prohibitive that interior random coils cannot occur. In fact, using $\delta(n)=$ $1 /(2 m n)^{\alpha}$ with $\alpha$ equal to $1.5,2$, and $\infty$ gives at least two significant figure agreement for $f_{\text {hd }}$. The absolute differences between the $\alpha=1.5$ and $\alpha=\infty$ result for $f_{\text {hd }}$ is less than or equal to 0.002 . For many values of $w$ agreement was even better. These differences in all cases would be indistinguishable on the scale of the curves drawn in Figure 4.

A study of $f_{\text {hd }}$ vs. $w$ shown in Figure 4 leads to the qualitative conclusion that loop entropy acts to decrease the effective value of $\sigma$ and thereby makes the likelihood of helix-coil boundaries less probable. Hence for a given $f_{\text {hd }}, w$ obtained from theory without loop entropy is less 


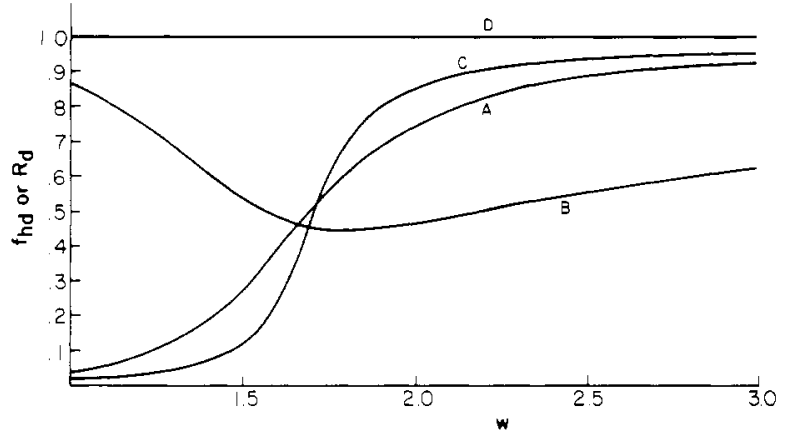

Figure 4. (A and B) $f_{\text {hd }}{ }^{0}$ and $R_{\mathrm{d}}{ }^{0}$, respectively, vs. $w$ calculated for the DNA-isomorphic model with neglect of loop entropy for a 284-residue, two-chain, coiled coil with $\sigma=7 \times 10^{-3}$ and $s=$ 0.94 in the $m=4$ coarse graining approximation. (C and D) $f_{\text {hd }}$ and $R_{\mathrm{d}}$, respectively, vs. $w$ calculated for the DNA-isomorphic model with $q_{\mathrm{I}}=0$ for a 284-residue two-chain, coiled coil with $\sigma=7 \times 10^{-3}$ and $s=0.94$ in the $m=4$ coarse graining approximation.

than or equal to $w$ obtained from theory with loop entropy if $f_{\mathrm{hd}} \leq 0.5$ and $w$ obtained from theory without loop entropy is greater than or equal to $w$ obtained from theory with loop entropy if $f_{\text {hd }} \geq 0.5$. A cursory glance at Figure 4 shows that differences in $w$ at the extremes of relatively high and low helix content can be appreciable.

A crucial qualitative observation emerges from the study of the DNA-isomorphic model: In this model any reasonable expression for the loop entropy will exclude interior random coil formation. We have chosen $C_{1}=1$ and set $d=0$ of eq I- 1 in the above as well as other calculations not shown here. (Note $d=0$ corresponds to a Gaussian chain.) In fact, we would expect $d>0$ and $C_{1} \ll 1$. Since even the simplest and least restrictive expression, that for Gaussian chains, acts to totally eliminate internal random coils, we conclude in general that in the interacting regions of the two-chain, coiled coil there are no interior loops of the $\mathrm{C}(1) \mathrm{C}(2)$ variety. A similar examination of typical values of $\delta_{2}(n)$ defined in eq I-2 leads us to believe that $\mathrm{C}(1) \mathrm{H}(2)$ interior loops are also unlikely to occur in the interacting portions of two-chain, coiled coils.

This conclusion, valid for the DNA-isomorphic model, does not, however, imply that there are no interior random coils in $\alpha$-helical, two-chain, coiled coils of moderate length even where loop entropy is important. In DNA double helices, as in the DNA-isomorphic model, both strands must be helical; i.e., if one of the two strands is nonhelical, the other strand must also be nonhelical. Otherwise stated, the statistical weight of a $\mathrm{C}(1) \mathrm{H}(2)$ block is zero. In two-chain, $\alpha$-helical, coiled coils, on the other hand, $\mathrm{C}(1) \mathrm{H}(2)$-type conformations are entirely possible (each of the strands in principle could exist as a single $\alpha$-helix). Consequently, while there are no interior coils in the interacting sequence of $\mathrm{H}(1) \mathrm{H}(2)$ block pairs, this does not preclude interior coils in the separated, noninteracting parts of the dimer, where they do not yield loops. (See Figures 1 and 3.) Every conformation of the molecule can contain a single interacting stretch of helical block pairs possibly preceded and followed by the noninteracting and separated portions of the two chains. Observe that in the separated strands there is no loop entropy. These are the important qualitative insights gained from the study of the DNA-isomorphic model. We next broaden the discussion to include these features peculiar to $\alpha$-helical coiled coils.

CC-Loops-Excluded Model. Before we develop the formalism that embodies the entire physics described above, it is instructive to consider the model in which $\mathrm{C}(1) \mathrm{C}(2)$ interior random coils cannot occur in the middle of a sequence of interacting helical blocks but in which
$\mathrm{C}(1) \mathrm{H}(2)$-type coils can occur. See Figure $3 \mathrm{C}$ for allowed and disallowed coil sequences. An interior $\mathrm{C}(1) \mathrm{C}(2)$ pair acts to partially separate the strands and thus turns off the helix-helix interaction. For convenience, we shall limit our discussion to a homopolymeric two-chain, coiled coil.

It is apparent that $q_{\mathrm{R}}(n)$ and $q_{\mathrm{L}}(n)$ are still given by eq IV-7 and eq IV-10 and IV-11, respectively, since these types of sequences do not contain any interior $\mathrm{C}(1) \mathrm{C}(2)$ random coiled block pairs; $q_{\mathrm{I}}\left(m_{\mathrm{I}}, n\right)$ is not, however, given by eq IV-13 when $w \neq 1$ and must be modified to correct for the possibility of one or more $\mathrm{C}(1) \mathrm{C}(2)$ block pairs occurring in blocks $m_{\mathrm{I}}+1, \ldots, m_{\mathrm{I}}+n$. When $w \neq 1$

$$
q_{\mathrm{I}}\left(m_{\mathrm{l}}, n\right)=\tau \mathscr{S} S M^{-n-1}\left\{q_{1}+q_{2}+q_{3}\right\} Z_{\mathrm{w}}^{-1}
$$

and

$$
\begin{array}{rl}
q_{1}=J * \mathrm{U}(w)^{m} & \mathrm{I} \mathrm{U}^{N-m_{\mathrm{I} J}}- \\
J^{*} \mathrm{U}(w)^{m_{\mathrm{I}}}\left[\begin{array}{ll}
0 & \tau S M^{n-1} \\
0 & S M^{n}
\end{array}\right] \mathrm{U}^{N \cdots m \mathrm{I}^{n} J}
\end{array}
$$

is the statistical weight of all the sequences in chain two in which helical blocks $1, \ldots, m_{1}$ interact with their counterparts on the adjacent chain but residues $m_{I}+1, \ldots, N$ cannot. The right-hand portion of the two chains starting at residues $m_{\mathrm{I}}+1$ are no longer side by side. The second set of matrix products in eq IV-19a removes the state

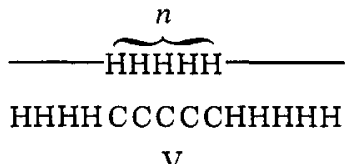

\section{$\mathrm{V}$}

which is incorrectly treated in $q_{1}$. This particular state has statistical weight $q_{3}$ (see below, eq IV-19c). Similarly

$$
\begin{aligned}
q_{2}=J^{*} \mathrm{U}^{m_{\mathrm{I}}+n} \mathrm{U}(w)^{N-m_{\mathrm{I}}-n} J- \\
\quad J^{*} \mathrm{U}^{m_{\mathrm{I}}}\left[\begin{array}{ll}
0 & \tau S M^{n-1} \\
0 & S M^{n}
\end{array}\right] \mathrm{U}(w)^{N-m_{\mathrm{I}}-n_{J}}
\end{aligned}
$$

$q_{2}$ is the statistical weight of all the sequences in chain two in which residues $1, \ldots, m_{\mathrm{I}}+n$ are noninteracting. Finally

$$
q_{3}=J * \mathrm{U}(w)^{m} \mathrm{I}\left[\begin{array}{ll}
0 & \tau S M^{n-1} \\
0 & S M
\end{array}\right] \mathrm{U}(w)^{N-m_{\mathrm{I}}-n} J \quad(\mathrm{IV}-19 \mathrm{c})
$$

is the statistical weight of sequence $\mathrm{V}$.

Note that eq IV-18 has introduced additional interior coil states relative to the case where loop entropy is neglected, eq IV-13.

We have calculated $f_{\text {hd }}$ and $R_{\mathrm{d}}$ as a function of $w$ for a homopolymeric two-chain, coiled coil in which $\sigma=1.5 \times$ $10^{-3}, s=0.94, N=71$, and $m=4$. We have chosen these parameters because they yield values of $R_{\mathrm{d}}{ }^{0}$, calculated with the neglect of loop entropy similar to that seen in tropomyosin (see Table III).

If loop entropy is neglected, $f_{\text {hd }}{ }^{0}$ and $R_{\mathrm{d}}{ }^{0}$ calculated via Poland's recursion relation method and via the matrix technique (eq $40 \mathrm{a}$ of ref 1) agree to within 0.02 unit or better. In the high and low helix content limit where interfaces of helices and coils are uncommon, agreement is much better and was within 0.002 unit.

Figure 5 displays $f_{\mathrm{hd}}{ }^{0}$ and $R_{\mathrm{d}}{ }^{0}$ as a function of $w$ in curves $A$ and $B$, respectively, and in which loop entropy has been left out. Curves $\mathrm{C}$ and $\mathrm{D}$ show $f_{\mathrm{hd}}$ and $R_{\mathrm{d}}$ vs. $w$ calculated by using $q_{\mathrm{I}}\left(m_{\mathrm{I}}, n\right)$ of eq IV-18 in which $\mathrm{C}(1) \mathrm{C}(2)$ coils are excluded from the interior of a interacting $\mathrm{H}(1) \mathrm{H}(2)$ sequence. 


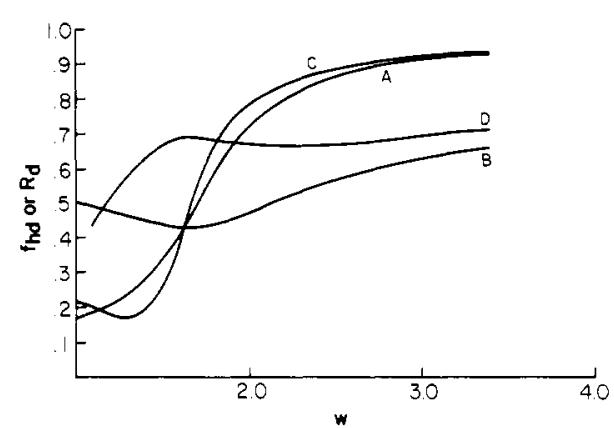

Figure 5. (A and B) $f_{\mathrm{hd}}{ }^{0}$ and $R_{\mathrm{d}}{ }^{0}$ vs. $w$ in homopolymeric dimer with $\sigma=1.5 \times 10^{-3}$ and $s=0.94$ in which loop entropy is neglected. (C and D) $f_{\text {hd }}$ and $R_{d}$ vs. $w$, respectively, in homopolymeric dimer with $\sigma=1.5 \times 10^{-3}$ and $s=0.94$ in the CC-loops-excluded model, i.e., in which $\mathrm{C}(1) \mathrm{C}(2)$ coils cannot occur in the middle of a sequence of interacting helical block pairs.

The behavior of $R_{\mathrm{d}}$ vs. $w$ in curve $\mathrm{D}$ can be rationalized as follows: At low helix content the dominant type of random coil is of the $\mathrm{C}(1) \mathrm{C}(2)$ type; it has statistical weight of 1 while a $\mathrm{CH}$ coil has statistical weight $s<1$. When interior $\mathrm{C}(1) \mathrm{C}(2)$ coils are excluded, there is essentially a single helical stretch in the interacting part of the dimer. Increasing $w$ acts to increase the mean length of the interacting helical stretch and thus must decrease the average number of interior random coils of the $\mathrm{C}(1) \mathrm{C}(2)$ type. $R_{\mathrm{d}}$ therefore initially increases as a function of $w$. However, as the mean helix content increases, so does the relative importance of $\mathrm{CH}$ coils, and thus $R_{\mathrm{d}}$ begins to level off and displays the usual concave-upward behavior (as in curve B) as a function of $w$.

At sufficiently small values of $w, q_{\mathrm{I}}$, defined in eq IV-18, introduces additional states whose total statistical weight may be larger than what would be seen if the chains were interacting. Consider the state HCH. Neglecting interior $\mathrm{CC}$ loops gives a statistical weight $\sigma^{2} s^{2} w^{2}$, while excluding $\mathrm{C}(1) \mathrm{C}(2)$ loops in an interacting block gives $2 \sigma^{2} s^{2} w$. Clearly, if $w<2$, the latter statistical weight dominates. At very low helix content then, $f_{\text {hd }}>f_{\text {hd }}{ }^{0}$. On the other hand, we have excluded $\mathrm{C}(1) \mathrm{C}(2)$ states from the interacting blocks and have thus made the transition more cooperative. Increasing $w$ causes the latter effect to dominate, and $f_{\text {hd }}{ }^{0}>f_{\text {hd }}$ if $f_{\text {hd }}{ }^{0}<50 \%$. Hence the $f_{\text {hd }}{ }^{0}$ curve must cross the $f_{\text {hd }}$ curve at very low helix content. As the helix content increases further, the curves across again. The minimum seen in Figure 5 is in reality an artifact of the Poland method (see discussion presented below). Note that the two curves do not cross at $f_{\text {hd }}{ }^{0}=0.50$ but rather at about 0.45 . This reflects the fact that while partial strand separation may be qualitatively viewed as reducing the mean $\sigma$, the analogy is not quantitative as states of the $\mathrm{CH}$ type are not precluded and $\mathrm{CC}$ coils are allowed to form on the ends.

Setting $\sigma=1 \times 10^{-4}, M=4$, and $N=71$, we find very close agreement between $f_{\mathrm{hd}}$ and $R_{\mathrm{d}}$ calculated with and without the exclusion of interior $\mathrm{C}(1) \mathrm{C}(2)$ coils. $\left(R_{\mathrm{d}}\right.$ exceeds 0.95 for all values of $w$.)

At sufficiently small $\sigma$, e.g., $1 \times 10^{-5}$, even in the absence of loop entropy, the Poland method gives a minimum in $f_{\text {hd }}$ and $Z_{\text {sd }}$ vs. $w$ at low helix content. Use of the matrix method gave a slightly increasing partition function and an ever so slightly increasing helix content throughout the same range of $w$. This reflects round off error inherent in the Poland method and is mentioned in Poland's appendix B. ${ }^{35}$ As we are interested in the regime where $f_{\text {hd }}$ $>50 \%$, these errors are unimportant and need not concern us further at this time (see point 2 of discussion following eq III-21).
Loops-Excluded Model. We are now in a position to address the maximum effect that loop entropy could have on the helical-coil transition in $\alpha$-helical, two-chain, coiled coils; i.e., we disallow $\mathrm{C}(1) \mathrm{C}(2)$ and $\mathrm{C}(1) \mathrm{H}(2)$ interior random coils between interacting helical block pairs. We shall refer to this model as the loops-excluded model. Thus in a given molecule there is only a single interacting helical stretch, and that part of the molecule is entirely helical. See Figure 3D. There may also be noninteracting helical stretches. This requires that we suitably modify $q_{\mathrm{R}}, q_{\mathrm{L}}$, and $q_{\mathrm{I}}$ to incorporate the aforementioned physics. We shall begin with the construction of $q_{R}$.

$\boldsymbol{q}_{\mathrm{R}}$ with Effect of Loop Entropy. Let $q_{\mathrm{R}}(N-n)$ be the statistical weight of a sequence of $N-n$ randomly coiled block pairs on the right end of chain one.

\section{$\overbrace{\mathrm{HHHHHCCCCC}}^{n} \overbrace{\text { CC }}^{N-n}$}

VI

The $n$th block on chain two may either be helical or randomly coiled. Let $r_{\mathrm{H}}(n)\left(r_{\mathrm{C}}(n)\right)$ be the total statistical weight of all the conformations in blocks $1, \ldots, n$ when the $n$th block on chain two is helical (randomly coiled). Then

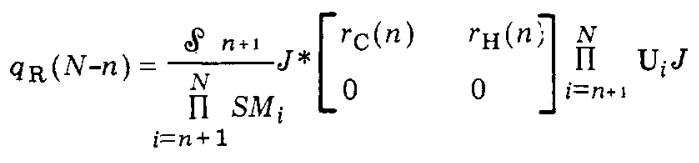
A.

Expressions for $r_{\mathrm{H}}(n)$ and $r_{\mathrm{C}}(n)$ are derived in Appendix

$\boldsymbol{q}_{\mathrm{L}}$ with the Effect of Loop Entropy. We now proceed to construct $q_{\mathrm{L}}(n)$, the statistical weight of chain one having $n$ randomly coiled blocks on the left-hand end of chain one (see sequence III). If $n \leq N-1$

$$
q_{\mathbf{L}}(n)=\frac{\tau_{n+1}}{\tau_{1}} J * \prod_{i=1}^{N} \mathbf{U}_{i}\left[\begin{array}{ll}
r_{\mathrm{CC}}(n) & r_{\mathrm{CH}}(n) \\
r_{\mathrm{H} \mathrm{C}}(n) & r_{\mathrm{H} \mathrm{H}}(n)
\end{array}\right] J /
$$

with $r_{\mathrm{C}}(N)$ and $r_{\mathrm{H}}(N)$ defined in eq A-10 and A-12, respectively. $r_{\alpha \beta}$ are the statistical weights associated with all the conformations of $n+1, \ldots, N$ blocks on chain two when the $n$th block on chain two is in conformation $\alpha$ and $(n+1)$ th block is in conformation $\beta . \alpha$ and $\beta$ may be either $\mathrm{C}$ or H. Explicit expressions for the $r_{\alpha \beta}$ are derived in Appendix B. Moreover

$q_{\mathrm{L}}(N)=\boldsymbol{J} * \prod_{i=1}^{N} \mathbf{U}_{i} \boldsymbol{J} /\left\{\tau_{1} \prod_{i=2}^{N} S M_{i}\left[r_{\mathrm{C}}(N)+r_{\mathrm{H}}(N)\right]\right\}$

$\boldsymbol{q}_{\mathrm{I}}$ with the Effect of Loop Entropy. Once $q_{\mathrm{R}}$ and $q_{\mathrm{L}}$ are known, it is straightforward to construct $q_{\mathrm{I}}\left(m_{\mathrm{I}}, n\right)$.

$$
\begin{aligned}
& q_{\mathrm{I}}\left(m_{\mathrm{I}}, n\right)= \\
& \tau_{m+n+1} \mathfrak{s}_{m+1}\left\{q_{1}+q_{2}\right\} /\left\{\prod_{i=m+1}^{m+n+1} S M_{i}\left[r_{\mathrm{C}}(N)+r_{\mathrm{H}}(N)\right]\right\}
\end{aligned}
$$

with

$$
q_{\mathrm{I}}=J *\left[\begin{array}{ll}
r_{\mathrm{C}}\left(m_{\mathrm{I}}\right) & r_{\mathrm{H}}\left(m_{\mathrm{I}}\right) \\
0 & 0
\end{array}\right] \prod_{i=m_{\mathrm{I}^{+1}}}^{N} U_{i} J
$$

and

$$
q_{2}=J * \prod_{i=1}^{m} \mathrm{U}_{i}\left[\begin{array}{ll}
r_{\mathrm{C} \mathrm{C}}\left(m_{\mathrm{T}}\right) & r_{\mathrm{CH}}\left(m_{\mathrm{T}}\right) \\
r_{\mathrm{HC}}\left(m_{\mathrm{T}}\right) & r_{\mathrm{HH}}\left(m_{\mathrm{T}}\right)
\end{array}\right] J
$$




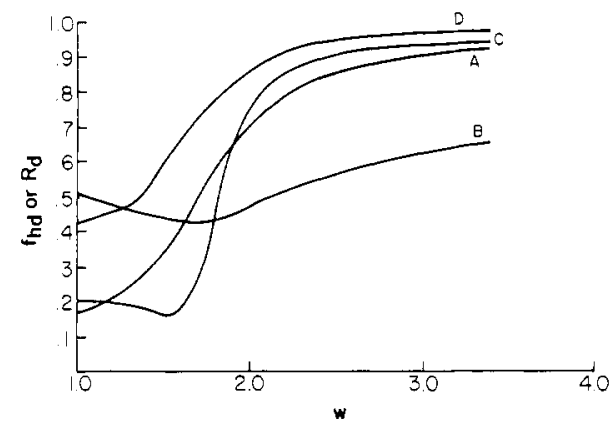

Figure 6. (A and B) $f_{\text {hd }}{ }^{0}$ and $R_{d}{ }^{0}$, respectively, vs. $w$ for a homopolymeric two-chain, coiled coil, in which loop entropy is ignored. (C and D) $f_{\text {hd }}$ and $R_{\mathrm{d}}$, respectively, vs. $w$ for the loops-excluded model in which loop entropy produces a single interacting helical stretch. In all cases $\sigma=1.5 \times 10^{-3}, s=0.94, N=71$, and $m=$ 4.

where $m_{\mathrm{T}}=m_{\mathrm{I}}+n\left(1 \leq m_{\mathrm{I}} \leq N-2,1 \leq n \leq N-m_{\mathrm{I}}-1\right.$. The $q_{1}$ term generates all those sequences on chains whose left-hand side contains interacting helical pairs (see eq IV-20, $q_{\mathrm{R}}$-type expression) and $q_{2}$ generates all those sequences on chains whose right-hand side contains the interacting helical pairs (see eq IV-21, $q_{\mathrm{L}}$-type expression).

Calculations on a Homopolymeric, Two-Chain, Coiled Coil. Taking $\sigma=10^{-5}, s=0.94, m=4$, and $N=$ 71 , we find virtually identical agreement for $f_{\text {hd }}$ vs. $w$ calculated with (using eq IV-20-23) and without the effect of loop entropy (using eq IV-7, $-10,-11$, and -13). Values of $\sigma=10^{-5}$ result in essentially no interior random coil formation and as required, the two treatments yield identical results.

In Figure 6 we have plotted $f_{\text {hd }}$ and $R_{\mathrm{d}}$ vs. $w$ for a coiled coil in which $\sigma=1.5 \times 10^{-3}, s=0.94, m=4$, and $N=71$. Curves $\mathrm{A}$ and $\mathrm{B}$ refer to $f_{\mathrm{hd}}{ }^{0}$ and $R_{\mathrm{d}}{ }^{0}$ vs. $w$ calculated with neglect of loop entropy. Curves $\mathrm{C}$ and $\mathrm{D}$ show $f_{\text {hd }}$ and $R_{\mathrm{d}}$ vs. $w$ calculated in the single interacting helix approximation, i.e., the loops-excluded model. Again we remind the reader that the minimum in $f_{\mathrm{hd}}$ is a result of round off error and should really be a plateau region. In the range of interest, $f_{\text {hd }}>50 \%$, the method is accurate. We call attention to the difference between curves $\mathrm{A}$ and $\mathrm{C}$ calculated with and without loop entropy. E.g., at $90 \%$ helix content the ratio of $w$ calculated without and with loop entropy is $2.9 / 2.4$, or about $100 \mathrm{cal} \cdot(\mathrm{mol} \text { of block pairs })^{-1}$. The difference between the two curves is greatest at moderately high and low helix contents. However, by use of the effective-decrease-in- $\sigma$ argument the curves in general must cross. Observe that the curves do not cross at $50 \%$ helix content. This reflects both the finite size of the chains and the difference between merely decreasing the value of $\sigma$ and requiring that a single interacting helical stretch exists in the interacting portion of the dimer. Thus, the values of $w$ required to yield a given $f_{\mathrm{hd}}$ will be larger calculated from a theory that includes loop entropy than from a theory without loop entropy at low helix content and smaller calculated from the theory including loop entropy than from a theory ignoring loop entropy at high helix content; the precise magnitude of the difference will depend on $\sigma$ and $N T$.

Observe that $R_{\mathrm{d}}$ vs. $w$ (curve D) calculated with the effect of loop entropy is now a monotonically increasing function of $w$. Since the mean helix content increases by increasing the mean length of single interacting helix stretch, the number of interior coils must decrease; interior coils are only allowed in the noninteracting pieces of the dimer. Hence as the helix content increases, $R_{\mathrm{d}}$ must also increase. The behavior of $R_{\mathrm{d}}$ vs. $w$ shown in Figure 6 is to be contrasted with that given in Figure 4 for the

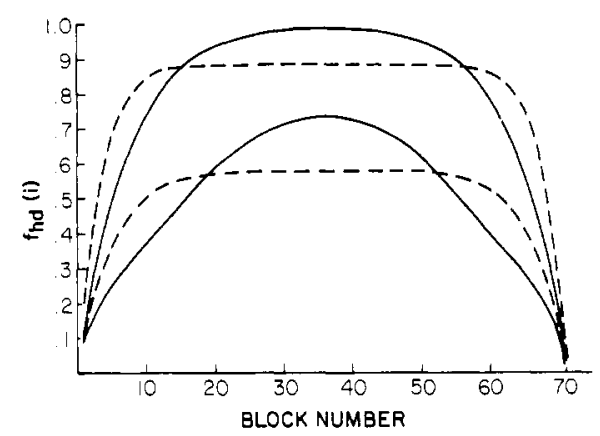

Figure 7. Bottom solid curve, helix probability profiles for a two-chain, coiled coil with $f_{\text {hd }}$ equal to 0.503 in which interior random coils in the interacting parts of the dimer are excluded, i.e., the loops-excluded model. Bottom dashed curve, helix probability profiles for a two-chain, coiled coil with $f_{\text {hd }}{ }^{0}$ equal to 0.502 and in which loop entropy has been ignored. Top solid curve, helix probability profile for a two-chain, coiled coil with $f_{\text {hd }}$ equal to 0.799 in which interior random coils in the interacting parts of the dimer are excluded, i.e., the loops-excluded model. Top dashed curve, helix probability profile for a two-chain, coiled coil with $f_{\text {hd }}{ }^{0}$ equal to 0.803 and in which loop entropy has been ignored. In all cases, $\sigma=1.5 \times 10^{-3}, s=0.94, m=4$, and $N=$ 71 .

DNA-isomorphic model. In the latter curve, $R_{\mathrm{d}}$ is essentially unity throughout the values of $w$ studied.

At a given mean helix content, loop entropy exerts an important influence on the helix probability profile as seen in Figure 7. In the lower set of curves $f_{\text {hd }}$ is about 0.50 and in the upper set $f_{\text {hd }}$ is about 0.80 . The dashed curves are calculated with neglect of loop entropy; the solid curves are calculated with the maximum effect of loop entropy (loops excluded) included. Loop entropy results in an increased maximum in the probability profile; concomitantly, the helix content near the ends is decreased. This is a natural consequence of the constraint that loop entropy produces a single interacting stretch in a given molecule.

In summary, the qualitative conclusions that emerge from the study of homopolymeric two-chain, coiled coils with loop entropy in the loops excluded model are as follows:

(1) The value of $w$ required for the same $f_{\text {hd }}$ with and without interior coils in the interacting helical stretches may differ substantially.

(2) $R_{\mathrm{d}}$ is a monotonic function of $w$; that is, the helix-coil transition involves melting from the ends of a single stretch of helical block pairs. Introduction of interior random coils at block $i$ serves to partially separate the strands elsewhere and effectively turns off the helix-helix interaction from block pair $i$ to the end of the molecule.

(3) The single interacting helix approximation that incorporates the effect of loop entropy into the partition function produces a higher maximum in the helix probability profile than where loop entropy is neglected. This observation is an immediate result of (2).

\section{Discussion of Results}

In this paper we have extended the theory of the helix-coil transition in $\alpha$-helical, two-chain, coiled coils to include the effects of loop entropy. For chains of moderate length and values of $\sigma$ where interior random coil formation is likely, loop entropy is so severe as to eliminate loops; i.e., it eliminates interior random coils from those sequences bounded by interacting (chain associated) helical sequences. Hence in chains in which loop entropy is important there is a single interacting helical stretch possibly preceded and followed by noninteracting blocks that may or may not be helical.

To estimate when loop entropy is important, one should 
calculate, with the neglect of loop entropy, $R_{\mathrm{d}}$, the ratio of number of random coil blocks that unwind from the ends to the total number of coiled blocks. If $R_{\mathrm{d}}$ is near unity, the contribution of loop entropy to the helix-coil transition will be negligible, and our original treatment which ignored loop entropy can be safely employed. Algorithms to calculate $R_{\mathrm{d}}$ have been presented.

In cases where loop entropy may be important, we have extended Poland's recursion relation method to incorporate the effect of loop entropy into our treatment. By suitable manipulation the expressions for $q_{\mathrm{R}}, q_{\mathrm{L}}$, and $q_{\mathrm{I}}$ have been reduced to, at worst, double sums over statistical weight matrix elements. In other words, we have developed a hybrid matrix-recursion relation technique that allows us to include correlations up to the total number of residues in the chain by summing over products of $2 \times$ 2 statistical weight matrices.

We remark here that it is possible to forego Poland's recursion relation method in its entirety and incorporate the single interacting helix approximation as a sum of products of $4 \times 4$ statistical weight matrices. This alternative and formally equivalent method will be developed in a forthcoming paper.

In addition, we shall apply the generalized Poland treatment to extract $w(T)$ from the thermal denaturation curves of rabbit $\alpha$-tropomyosin at acidic and near-neutral $\mathrm{pH}$. Clearly, on the basis of Table III loop entropy may be important. $w(T)$ extracted from experiment with a theory that includes the effect of loop entropy will presumably be a measure of the mean helix-helix interaction alone. A detailed knowledge of the factors constituting $w$ is important, for only then can we elucidate the nature of the interhelix stability in $\alpha$-helical, two-chain, coiled coils. That is, after all, the primary motivation of this avenue of research.

Acknowledgment is made to the donors of the Petroleum Research Fund, administered by the American Chemical Society, for support of this research. Special thanks are due to Professor Alfred Holtzer for his many stimulating discussions and his critical reading of the manuscript. Useful discussions with Professors Wayne Mattice and Robert Yaris are gratefully acknowledged.

\section{Appendix A. Construction of $\boldsymbol{r}_{\mathrm{H}}(\boldsymbol{n})$ and $\boldsymbol{r}_{\mathrm{C}}(\boldsymbol{n})$}

We begin with the calculation of $r_{\mathrm{H}}(n)$. The $n$th block on chain two may be part of an interacting helical stretch of length $l \leq n$ or, if $l<n$, the $n$th helical residue may be noninteracting with the block on the adjacent chain. This occurs because internal $\mathrm{H}(1) \mathrm{C}(2)$ blocks separate the two strands and act to turn off the helix-helix interaction. If the $n$th block on chain two is helical and $w \neq 1$, let us denote the statistical weight of all such interacting conformations by $r_{\mathrm{H}}{ }^{\mathrm{i}}(n)$. On the other hand, if the $n$th block on chain two is helical and has been preceded by a randomly coiled block, it possibly cannot interact with the $n$th helical block on chain one and therefore has $w=1$. Let us define the statistical weight of all such sequences by $r_{\mathrm{H}}{ }^{0}(n)$.

Now the statistical weight of all conformations of blocks $1, \ldots, n$ on chain two having the $n$th block helical and interacting with the $n$th block on chain one is

$$
\begin{gathered}
r_{\mathrm{H}}^{\mathrm{i}}(1)=\tau_{1} w \\
r_{\mathrm{H}}^{\mathrm{i}}(2)=\tau_{2} w+\tau_{1} S M_{2} w^{2}
\end{gathered}
$$

and for all $n \geq 3$

$$
\begin{aligned}
& r_{\mathrm{H}}^{\mathrm{i}}(n)= \sum_{i=1}^{n-2} J * \prod_{i=1}^{n-l-1} \mathbf{U}_{i}\left[\begin{array}{lr}
1 & 0 \\
\mathcal{S}_{n-l} & 0
\end{array}\right]\left[\begin{array}{ll}
0 & \tau_{n-l} w^{l} \prod_{i=1}^{n-2} S M_{n-i+1} \\
0 & 0
\end{array}\right] J+ \\
& \tau_{2} w^{n-1} \prod_{i=1}^{n-2} S M_{n-i+1}+\tau_{1} \prod_{i=1}^{n-1} S M_{i} w^{n}
\end{aligned}
$$

For a homopolymer we can write

$$
\begin{aligned}
r_{\mathrm{H}}^{\mathrm{i}}(n)=\sum_{l=1}^{n-2} \tau w^{l} S M^{l-1}\left\{U_{11}{ }^{n-l-1}+\mathcal{S} U_{12}{ }^{n-l-1}\right\}+ \\
\tau w^{n-1} S M^{n-2}+\tau w^{n} S M^{n-1} \text { (A-4a) }
\end{aligned}
$$

wherein

$$
\mathbf{U}^{n-l-1}=\prod_{i=1}^{n-l-1} \mathbf{U}_{i}
$$

Furthermore, if the $n$th block on chain two is helical but noninteracting with the $n$th block on chain one, we have

$$
\begin{aligned}
& r_{H^{0}}{ }^{0}(1)=0 \\
& r_{H}{ }^{0}(2)=0
\end{aligned}
$$

that is, at least one helical block in the dimer is required to interact with the corresponding block on the other chain. If $n \geq 3$, the maximum length of the interacting helical sequence is $l=n-2$, and the $(n-1)$ th block must be randomly coiled. Hence

$$
\begin{array}{r}
r_{\mathrm{H}}{ }^{0}(n)=\sum_{l=1}^{n-2} \sum_{j=0}^{n-2-l} J^{j} \prod_{i=1}^{j-1} \mathrm{U}_{i}\left[\begin{array}{ll}
1 & 0 \\
\mathcal{S}_{j} & 0
\end{array}\right]\left[\begin{array}{lll}
0 & \tau_{j+1} w^{l} \prod_{i=2}^{l} & S M_{j+i} \\
0 & 0 &
\end{array}\right] . \\
\\
{\left[\begin{array}{ll}
0 & 0 \\
\mathcal{S}_{j+l+1} & 0
\end{array}\right] \prod_{i=j+l+2}^{n-1} \mathrm{U}_{i}\left[\begin{array}{ll}
0 & \tau_{n} \\
0 & S M_{n}
\end{array}\right] J(\mathrm{~A}-7)}
\end{array}
$$

Here, we use the convention that $\prod_{i=\beta}^{\alpha} \mathbf{U}_{i}=\mathbf{E}$ if $\beta>\alpha$ In the homopolymeric limit we have

$$
\begin{array}{r}
r_{\mathrm{H}_{i}}{ }^{0}(n)=\sum_{l=1}^{n-2} \sum_{j=0}^{n-2-l} \tau w^{l} S M^{l-1} \mathfrak{S}\left[U_{11^{j-1}}+U_{12}{ }^{j-1} \mathcal{S}\right] \times \\
{\left[\tau U_{11}{ }^{n-j-l-2}+S M U_{12}{ }^{n-j-l-2}\right]}
\end{array}
$$

with

$$
\begin{gathered}
U_{i j}{ }^{\alpha}=\delta_{i j} \quad \text { if } \alpha \leq 0 \\
U_{i j}{ }^{\alpha}=\left(\prod_{l=1}^{\alpha} \mathbf{U}_{l}\right)_{i j} \quad \text { if } \alpha>0
\end{gathered}
$$

Now $r_{\mathrm{H}}(n)$ is given by

$$
r_{\mathrm{H}}(n)=r_{\mathrm{H}}^{\mathrm{i}}(n)+r_{\mathrm{H}}^{0}(n)
$$

with $r_{\mathrm{H}}^{\mathrm{i}}(n)$ given by eq A-1 to A-3 and $r_{\mathrm{H}}{ }^{0}(n)$ given by eq A-5 to A-7. The statistical weight of blocks $1, \ldots, n$ on chain two having the $n$th block in the random coil state, $r_{\mathrm{C}}(n)$, is given by

$$
r_{\mathrm{C}}(1)=1
$$

and for $n>1$

$$
\begin{array}{r}
r_{\mathrm{C}}(n)=1+\sum_{l=1}^{n-1} \sum_{j=0}^{n-1}-l \prod_{i=1}^{j-1} \mathrm{U}_{i}\left[\begin{array}{ll}
1 & 0 \\
\mathcal{S}_{j} & 0
\end{array}\right]\left[\begin{array}{ll}
0 & \tau_{j+1} w^{l} \prod_{i=2}^{l} S M_{j+i} \\
0 & 0
\end{array}\right] . \\
{\left[\begin{array}{ll}
0 & 0 \\
\mathfrak{S}_{j+l+1} & 0
\end{array}\right] \prod_{i=j+l+2}^{n-1} \mathrm{U}_{i}\left[\begin{array}{ll}
1 & 0 \\
\mathfrak{S}_{n} & 0
\end{array}\right] J(\mathrm{~A}-12)}
\end{array}
$$

In $r_{\mathrm{C}}(n)$ the $n$th unit is by definition in a random coil state 
and the helix may be up to $n-1$ blocks in length.

Suppose the chains are homopolymeric; then

$r_{\mathrm{C}_{i}}(n)=1+\sum_{l=1}^{n-1} \sum_{j=0}^{n-1-l} \tau w^{l} S M^{l-1} \mathfrak{S}_{j+l+1}\left[U_{11}{ }^{j-1}+\mathcal{S} U_{12}{ }^{j-1}\right] \times$
$\left[U_{11^{n-j-l-2}}+\mathcal{S} U_{12^{n-j-l-2}}\right](\mathrm{A}-1$

where $U^{\alpha}$ is defined by eq A-9a and A-9b.

\section{Appendix B. Calculation of $\boldsymbol{r}_{\alpha \beta}$}

Let us start with the calculation of $r_{\mathrm{CH}}$, the statistical weight of all sequences in blocks $n+1, \ldots, N$ on chain two where the $n$th block is randomly coiled and the $(n+1)$ th block is helical.

$$
r_{\mathrm{CH}}(n)=r_{\mathrm{CH}^{\mathrm{i}}}{ }^{\mathrm{i}}(n)+r_{\mathrm{CH}^{0}}{ }^{0}(n)
$$

That is, the $(n+1)$ th helical block may be interacting or noninteracting with the $(n+1)$ th block on the chain one. $r_{\mathrm{CH}}{ }^{\mathrm{i}}(n)$ sums all statistical weights of blocks $n+1, \ldots, N$ associated with an interacting $n+1$ block and $r_{\mathrm{CH}}{ }^{0}(n)$ sums all the statistical weights of blocks $n+1, \ldots, N$ having a noninteracting $n+1$ helical block. Clearly

$$
\begin{gathered}
r_{\mathrm{CH}}^{\mathrm{i}}(N-1)=\tau_{N} w \\
r_{\mathrm{CH}^{\mathrm{i}}}(N-2)=\tau_{N-1} w_{N}+\tau_{N-1} S M_{N} w^{2}
\end{gathered}
$$

and for $n<N-2$

$$
\sum_{l=1}^{r_{\mathrm{CH}}{ }^{\mathrm{i}}(n)=} J^{N-n-1} J^{*}\left[\begin{array}{ll}
0 & \tau_{n+1} w^{l} \prod_{i=2}^{l} S M_{i} \\
0 & 0
\end{array}\right]\left[\begin{array}{lr}
0 & 0 \\
\mathcal{S}_{n+l+1} & 0
\end{array}\right] \prod_{i=n+l+2}^{N} \mathrm{U}_{i} J+
$$

Observe that the maximum length of an interacting helix is $N-n$. We use the convention $\prod_{i=\beta}^{\alpha} \mathbf{U}_{i}=\mathbf{E}$ if $\beta>\alpha$.

In the homopolymeric limit, eq B-4 may be simplified to yield

$$
\begin{aligned}
& r_{\mathrm{CH}_{i}}{ }^{\mathrm{j}}(n)= \\
& \sum_{l=1}^{N-n-1} \tau w^{l} S M^{l-1} \mathcal{S}\left\{U_{11}{ }^{N-n-l-1}+U_{\left.12^{N-n-l-1}\right\}}+\tau S M^{N-n-1} w^{N-n}\right.
\end{aligned}
$$

wherein $\mathbf{U}^{\alpha}$ is defined by eq $A-9 a$ and $A-9 b$.

Now suppose residue $n+1$ on chain two is part of the noninteracting $\mathrm{HH}$ block pair. The maximum length of the interacting helix is $N-n-2$ (block $n+2$ must be a random coil). Thus we can write

$$
r_{\mathrm{CH}}{ }^{0}(N-2)=r_{\mathrm{CH}^{0}}{ }^{0}(N-1)=0
$$

and when $n<N-2$

$$
\begin{aligned}
& r_{\mathrm{CH}}{ }^{0}(n)=\sum_{l=1}^{N-n-2} \sum_{j=0}^{N-n-2-l} J^{*}\left[\begin{array}{ll}
0 & \tau_{n+1} \\
0 & 0
\end{array}\right] \mathrm{U}_{n+2} \prod_{i=1}^{j} \mathrm{U}_{n+j+2} \cdot \\
& {\left[\begin{array}{ll}
0 & \tau_{n+j+3} \prod_{i=2}^{l} S M_{n+j+2+l} w^{l} \\
0 & 0
\end{array}\right]\left[\begin{array}{ll}
0 & 0 \\
\mathcal{S}_{n+j+3+l} & (
\end{array}\right] \underset{i=n+4+j+l}{\prod_{i}} \mathrm{U}_{i} J}
\end{aligned}
$$

where we again use the convention $\prod_{i=\beta}^{\alpha} \mathbf{U}_{i}=\mathbf{E}$ if $\beta>\alpha$.

Now if the chain is homopolymeric eq B-7 reduces to

$$
\begin{aligned}
r_{\mathrm{CH}_{i}}{ }^{\circ}(n)= & \sum_{l=1}^{N-n-2} \sum_{j=0}^{N-n-2-l} \tau^{2} w^{l} S M^{l-1} \mathcal{e}^{\alpha}{ }_{n+j+3+l} U_{21}{ }^{j+1} \times \\
& {\left[U_{11}{ }^{N-n-j-3-l}+U_{12}{ }^{N-n-j-3-}\right] }
\end{aligned}
$$

wherein

$$
\begin{gathered}
\mathcal{f}_{n+j+3+l}^{\alpha}=\mathcal{S}_{n+j+3+l} \quad \text { if } n+j+3+l \leq N \\
\mathcal{S}_{n+j+3+l}^{\alpha}=1 \quad \text { if } n+j+3+l>N
\end{gathered}
$$

and $\mathrm{U}^{\alpha}$ is defined in eq A-9a and A-9b.

In similar fashion we proceed to calculate $r_{\mathrm{HH}}(n)$, the total statistical weight of all the conformations of blocks $n+1, \ldots, N$ on chain two when blocks $n$ and $n+1$ are both helical. Set

$$
r_{\mathrm{HH}}(n)=r_{\mathrm{HH}^{\mathrm{i}}}(n)+r_{\mathrm{HH}}{ }^{0}(n)
$$

in which $r_{\mathrm{HH}}{ }^{\mathrm{i}}(n)\left(r_{\mathrm{HH}}{ }^{0}(n)\right)$ is the statistical weight appropriate when $n+1$ block pairs on chain one and two are interacting (noninteracting).

$$
\begin{gathered}
r_{\mathrm{HH}}(N-1)=S M_{N} w \\
r_{\mathrm{HH}}(N-2)=S M_{N-1} \oint_{N} w+S M_{N-1} S M_{N} w
\end{gathered}
$$

and for all $n<N-2$

$$
\begin{aligned}
r_{\mathrm{HH}}{ }^{\mathrm{i}}(n) & =\sum_{l=1}^{N-n-1} J^{*} \mathrm{t}\left[\begin{array}{ll}
0 & 0 \\
0 & w^{l} \prod_{i=1}^{l} S M_{n+i}
\end{array}\right] \cdot \\
& {\left[\begin{array}{ll}
0 & 0 \\
\mathfrak{S}_{n+l+1} & 0
\end{array}\right] \underset{i=n+l+2}{N} \mathrm{U}_{i} J+w^{N-n} \prod_{i=n+1}^{N} S M_{i} }
\end{aligned}
$$

where the usual convention $\prod_{i=\beta}^{\alpha} \mathbf{U}_{i}=\mathbf{E}$ if $\beta>\alpha$ has been adopted and $\boldsymbol{J}_{\mathrm{t}}=$ Row $(0,1)$.

Now if all the blocks are identical, eq B-12 becomes

$$
\begin{aligned}
& r_{\mathrm{HH}_{i}}^{\mathrm{i}}(n)= \\
& \quad \sum_{l=1}^{N-n-1} S M^{l} w^{l} \oint_{n+l+1}\left[U_{11}{ }^{N-n-l-1}+U_{12}{ }^{N-n-l-1}\right]+S M^{N-n} w^{n}
\end{aligned}
$$

and $\mathrm{U}^{\alpha}$ has been given in eq $\mathrm{A}-9 \mathrm{a}$ and $\mathrm{A}-9 \mathrm{~b}$. Moreover

$$
r_{\mathrm{HH}}{ }^{0}(N-1)=r_{\mathrm{HH}}{ }^{0}(N-2)=0
$$

If $n<N-2$

$$
\begin{aligned}
r_{\mathrm{HH}}{ }^{0}(n) & \sum_{l=1}^{N-n-2} \sum_{j=0}^{N-n-2-l} J^{*}{ }_{\mathrm{t}} \bullet \\
& {\left[\begin{array}{ll}
0 & 0 \\
0 & S M_{n+1}
\end{array}\right] \prod_{i=0}^{j} \mathrm{U}_{n+2+i}\left[\begin{array}{lll}
0 & \tau_{n+3} w^{l} \prod_{i=2}^{l} S M_{n+2+j+i} \\
0 & 0 &
\end{array}\right] . } \\
& {\left[\begin{array}{lll}
0 & & 0 \\
\mathscr{S}_{n+3+j+l} & 0
\end{array}\right] \prod_{i=n+4+j+l}^{N} \mathrm{U}_{i} J(B-15) }
\end{aligned}
$$

with the usual convention $\prod_{i=\beta}^{\alpha} \mathbf{U}_{i}=\mathbf{E}$ if $\alpha<\beta$

Note that the maximum length of an interacting helical stretch when the $(n+1)$ th block on chain two is helical but noninteracting with block $n+1$ on chain one is $N-$ $n-2$.

In the limit of a homopolymer we have

$r_{\mathrm{HH}_{i}}{ }^{0}(n)=\sum_{l=1}^{N-n-2} \sum_{j=0}^{N-n-2-l} \tau w^{l} S M^{l} \mathcal{S}^{\alpha}{ }_{n+j+3+l} U_{21}{ }^{j+1}\left[U_{11}{ }^{N-n-j-l-3}\right.$
$\left.+U_{12}{ }^{N-n-j-l-3}\right]$ (B-16)

with $\mathbf{U}^{\alpha}$ given in eq A-9a and $\mathrm{A}-9 \mathrm{~b}$ and $\mathcal{S}^{\alpha}{ }_{n+j+3+l}$ defined in eq B-8b.

$r_{\mathrm{CC}}(n)$, the statistical weight of all conformations of residues $n+1, \ldots, N$ on chain two when the $n$th and $(n$ +1 )th blocks are randomly coiled, is for $n=N-1$

$$
r_{\mathrm{CC}}(N-1)=1
$$


and for $n<N-1$

$$
\begin{aligned}
& r_{\mathrm{CC}}(n)=1+\sum_{l=1}^{N-n-1} J^{*}\left[\begin{array}{ll}
1 & 0 \\
0 & 0
\end{array}\right]_{i=1}^{j} \mathrm{U}_{n+i+1} \cdot \\
& {\left[\begin{array}{ll}
0 \tau_{n+j+2} i^{l} \prod_{i=2}^{l} S M_{n+j+i+1} \\
0 & 0
\end{array}\right]\left[\begin{array}{ll}
0 & 0 \\
\mathcal{S} \alpha_{n+j+2+l} & 0
\end{array}\right] \underset{i=n+j+3+l}{N} \mathrm{U}_{i} J}
\end{aligned}
$$

wherein $\prod_{i=\beta}^{\alpha} \mathbf{U}_{i}=\mathbf{E}$ if $\alpha<\beta$ and $\rho^{\alpha}{ }_{n+j+2+l}=\rho_{n+j+2+l}$ if $n$ $+j+2+l \leq N$ and equals one otherwise: the " 1 " in eq B-18 is the statistical weight of the $N-n$ block completely randomly coiled state.

For a homopolymer we have

$$
\begin{aligned}
& r_{\mathrm{CC}}(n)= \\
& 1+\sum_{l=1}^{N-n-1} \tau w^{l} S M^{l} \rho^{\alpha}{ }_{n+j+2+l} U_{11}{ }^{j}\left[U_{11}{ }^{N-n-j-2-l}+U_{12}{ }^{N-n-j-2-l}\right]
\end{aligned}
$$

wherein $\mathbf{U}^{\alpha}$ is, as previously, given in eq A-9a and A-9b.

Finally, if $r_{\mathrm{HC}}$ is the statistical weight of all the allowed conformations of blocks $n+1, \ldots, N$ on chain two when block $n$ is helical and block $n+1$ is a random coil, it can be shown that

$$
r_{\mathrm{HC}}(n)=\mathfrak{s}_{n+1} r_{\mathrm{CC}}(n)
$$

\section{References and Notes}

(1) Skolnick, J.; Holtzer, A. Macromolecules 1982, 15, 303.

(2) (a) Skolnick, J.; Holtzer, A. Macromolecules 1982, 15, 812. (b) Holtzer, M. E.; Holtzer, A.; Skolnick, J. Macromolecules 1983, 16, 173 .

(3) Ananthanarayanan, V. S.; Andreatta, R. H.; Poland, D.; Scheraga, H. A. Macromolecules 1971, 4, 417.

(4) Platzer, K. E. B.; Ananthanarayanan, V. S.; Andreatta, R. H.; Scheraga, H. A. Macromolecules 1972, 5, 177.

(5) Alter, J. E.; Taylor, G. T.; Scheraga, H. A. Macromolecules $1972,5,739$.

(6) Van Wart, H. E.; Taylor, G. T.; Scheraga, H. A. Macromolecules $1973,6,266$.

(7) Alter, J. E.; Andreatta, R. H.; Taylor, G. T.; Scheraga, H. A. Macromolecules 1973, 6, 564 .
(8) Maxfield, F. R.; Alter, J. E.; Taylor, G. T.; Scheraga, H. A Macromolecules 1975, 8, 479.

(9) Scheule, R. K.; Cardinaux, F.; Taylor, G. T.; Scheraga, H. A. Macromolecules 1976, 9, 23.

(10) Dygert, M. K.; Taylor, G. T.; Cardinaux, F.; Scheraga, H. A Macromolecules 1976, 9, 794 .

(11) Matheson, R. R.; Nemenoff, R. A.; Cardinaux, F.; Scheraga, H A. Biopolymers $1977,16,1567$.

(12) van Nispen, J. W.; Hill, D. J.; Scheraga, H. A. Biopolymers $1977,16,1587$.

(13) Hill, D. J.; Cardinaux, F.; Scheraga, H. A. Biopolymers 1977, $16,2447$.

(14) Konishi, Y.; van Nispen, J. W.; Davenport, G.; Scheraga, H. A. Macromolecules 1977, 10, 1264.

(15) Kobayashi, Y.; Cardinaux, F.; Zweifel, B. O.; Scheraga, H. A Macromolecules 1977, 10, 1271.

(16) Hecht, M. H.; Zweifel, B. O.; Scheraga, H. A. Macromolecules $1978,11,545$.

(17) Hodges, R.; Saund, A.; Chung, P.; St.-Pierre, S.; Reid, R. J. Biol. Chem. 1981, 256, 1214.

(18) Poland, D. C.; Scheraga, H. A. Biopolymers 1965, 3, 305.

(19) Schellman, J. A. C. R. Trav. Lab. Carlsberg., Ser. Chim. 1955 , $29,230$.

(20) Flory, P. J. J. Am. Chem. Soc. 1956, 78, 5222.

(21) Jacobson, H.; Stockmayer, W. H. J. Chem. Phys. 1950, 18, 1600.

(22) Poland, D.; Scheraga, H. A. "Theory of Helix-Coil Transitions in Biopolymers"; Academic Press: New York, 1970.

(23) (a) Fixman, M.; Freire, J. J. Biopolymers 1977, 16, 2693. (b) de Gennes, P.-G. "Scaling Concepts in Polymer Physics"; Cornell University Press: Ithaca, NY, 1979.

(24) Zimm, B.; Bragg, J. J. Chem. Phys. 1959, 31, 526.

(25) Mattice, W. L.; Skolnick, J. Macromolecules 1982, 15, 1088.

(26) Hodges, R.; Sodek, J.; Smillie, L.; Jurasek, L. Cold Spring Harbor Symp. Quant. Biol. 1972, 37, 299.

(27) McLachlan, A.; Stewart, M. J. Mol. Biol. 1975, 98, 293.

(28) Stone, D.; Smillie, L. J. Biol. Chem. 1978, 253, 1137.

(29) Mak, A.; Lewis, W.; Smillie, L. FEBS Lett. 1979, 105, 232

(30) Crothers, D. M.; Kallenbach, N. R. J. Chem. Phys. 1966, 45, 917.

(31) Flory, P. "Statistical Mechanics of Chain Molecules"; Wiley: New York, 1969; Chapter 3.

(32) Holtzer, M. E.; Holtzer, A.; Skolnick, J. Macromolecules 1983 $16,462$.

(33) Lifson, S.; Roig, A. J. Chem. Phys. 1961, 34, 1963.

(34) Pato, M.; Mak, A.: Smillie, L. J. Biol. Chem. 1981, 256, 593

(35) Poland, D. Biopolymers $1974,13,1859$. We shall refer to equations in Poland's paper by the prefix P.

\title{
Theory of Phase Equilibria in Systems Containing Block Copolymers
}

\author{
Kin Ming Hong and Jaan Noolandi* \\ Xerox Research Centre of Canada, 2480 Dunwin Drive, \\ Mississauga, Ontario, Canada L5L 1J9. Received September 30, 1982
}

\begin{abstract}
A theory for inhomogeneous multicomponent polymer systems developed earlier by the authors is simplified for the case where the inhomogeneity is weak, and this theory is used to study the phase diagrams of a mixture of block copolymers, homopolymers, and solvents. The free energy of the system is expressed as a functional of the deviations in the concentration profiles from their homogeneous values, and a perturbation expansion up to fourth order in the fluctuations is carried out. For a mixture of a block copolymer and a nonselective solvent a simple expression is derived for the inhomogeneous free energy term, and some typical phase diagrams are calculated. The existence of eutectic points, similar to those studied in metallurgy, is demonstrated. Phase diagrams for block copolymer-homopolymer systems are also discussed, and homopolymer-induced mesophase formation is predicted. The periodicity of the lamellar structure with varying homopolymer concentration is calculated, and the density profiles near demixing are shown.
\end{abstract}

\section{Introduction}

A polymeric blend containing block copolymers can exhibit a macroscopic phase separation as well as a microscopic one. These polymeric alloys have been intensively studied in recent years because of their remarkable structural and mechanical properties. ${ }^{1-3}$ The phase sep- aration behavior of such systems has also been a problem of longstanding interest to theorists. ${ }^{4-15}$ Krause $^{4}$ has adopted a thermodynamic approach, and described the phase separations in macroscopic terms. Meier, ${ }^{5,6}$ on the other hand, has studied the microdomain formation from a more microscopic point of view, and on the basis of these 